\title{
Meflin-positive cancer-associated fibroblasts enhance tumor response to immune checkpoint blockade
}

\author{
Yuki Miyai, (10, Daisuke Sugiyama ${ }^{3}$, Tetsunari Hase ${ }^{4} \mathbb{\infty}$, Naoya Asai ${ }^{1}$, Tetsuro Taki ${ }^{1}$, Kazuki Nishida ${ }^{5} \mathbb{D}$, Takayuki Fukui ${ }^{6}$, \\ Toyofumi Fengshi Chen-Yoshikawa ${ }^{6}$, Hiroki Kobayashi ${ }^{1} \mathbb{0}$, Shinji Miii, Yukihiro Shiraki ${ }^{1} \mathbb{0}$, Yoshinori Hasegawa ${ }^{4}$, \\ Hiroyoshi Nishikawa ${ }^{3,7}$ (D), Yuichi Ando², Masahide Takahashi', Atsushi Enomoto ${ }^{1}$ (1)
}

\begin{abstract}
Cancer-associated fibroblasts (CAFs) are an integral component of the tumor microenvironment (TME). Most CAFs shape the TME toward an immunosuppressive milieu and attenuate the efficacy of immune checkpoint blockade (ICB) therapy. However, the detailed mechanism of how heterogeneous CAFs regulate tumor response to ICB therapy has not been defined. Here, we show that a recently defined CAF subset characterized by the expression of Meflin, a glycosylphosphatidylinositol-anchored protein marker of mesenchymal stromal/stem cells, is associated with survival and favorable therapeutic response to ICB monotherapy in patients with non-small cell lung cancer (NSCLC). The prevalence of Meflin-positive CAFs was positively correlated with CD4-positive T-cell infiltration and vascularization within non-small cell lung cancer tumors. Meflin deficiency and CAF-specific Meflin overexpression resulted in defective and enhanced ICB therapy responses in syngeneic tumors in mice, respectively. These findings suggest the presence of a CAF subset that promotes ICB therapy efficacy, which adds to our understanding of CAF functions and heterogeneity.
\end{abstract}

DOI 10.26508/lsa.202101230 | Received 8 September 2021 | Revised 18 February 2022 | Accepted 18 February 2022 | Published online 1 March 2022

\section{Introduction}

As immune checkpoint blockade (ICB) therapy is emerging as a promising treatment strategy for a wide range of cancers, understanding the mechanisms underlying tumor immunity and identifying biomarkers that predict patient outcomes has been a focus of cancer research (Rizvi et al, 2015; Kumagai et al, 2020a, 2020b; House et al, 2020; Mager et al, 2020; Smith et al, 2021). The intrinsic properties of tumor cells, mutational burdens, and their interactions with host immune cells are critical for the efficacy of ICB (Rizvi et al, 2015; Kumagai et al, 2020a, 2020b). However, only a subset of patients with cancer benefits from ICB therapy, and patients exhibit a variable response to it across cancer types (Carbognin et al, 2015). Therefore, additional studies are needed to understand the influence of the tumor microenvironment (TME) and its constituents on ICB therapy response.

Cancer-associated fibroblasts (CAFs) are a major component of the TME and accumulate in the tumor stroma across multiple cancers (Kalluri, 2016; Kobayashi et al, 2019; Miyai et al, 2020; Piersma et al, 2020). Recent single-cell sequencing analyses have revealed that CAFs can be segregated into several clusters based on their transcriptome (Öhlund et al, 2017; Costa et al, 2018; Lambrechts et al, 2018; Elyada et al, 2019; Kieffer et al, 2020). Major defined subpopulations of CAFs, referred to as myofibroblastic CAFs (myCAFs), inflammatory CAFs (iCAFs), and antigen-presenting CAFs (apCAFs), were first described in pancreatic cancer (Öhlund et al, 2017; Elyada et al, 2019). Single-cell analysis of tumor stroma provided evidence of similar CAF populations in other cancer types, such as breast and lung cancer (Costa et al, 2018; Lambrechts et al, 2018; Kieffer et al, 2020). CAFs are now understood to be a major source of immunosuppressive activity in the TME (Barrett \& Puré, 2020; Baker et al, 2021). A pioneering study showed that the CAF-S1 subset, which is characterized by $\alpha$-smooth muscle actin ( $\alpha$-SMA) and fibroblast activation protein (FAP) expression, is crucial for the induction of regulatory $T$ cells to promote cancer progression and immunotherapy resistance (Costa et al, 2018; Kieffer et al, 2020). Another study revealed that the infiltration of CAFs expressing leucine-rich repeat-containing 15 (LRRC15), whose expression was induced by TGF- $\beta$, correlated with poor response to ICB therapy across multiple cancer types (Dominguez et al, 2020). Other studies

\footnotetext{
${ }^{1}$ Department of Pathology, Nagoya University Hospital, Nagoya, Japan ${ }^{2}$ Department of Clinical Oncology and Chemotherapy, Nagoya University Hospital, Nagoya, Japan ${ }^{3}$ Department of Immunology, Nagoya University Hospital, Nagoya, Japan ${ }^{4}$ Department of Respiratory Medicine, Nagoya University Hospital, Nagoya, Japan ${ }^{5}$ Center for Advanced Medicine and Clinical Research, Nagoya University Hospital, Nagoya, Japan ${ }^{6}$ Department of Thoracic Surgery, Nagoya University Graduate School of Medicine, Nagoya, Japan ${ }^{7}$ Division of Cancer Immunology, Research Institute/Exploratory Oncology Research and Clinical Trial Center (EPOC), National Cancer Center, Tokyo, Japan
}

Correspondence: enomoto@iar.nagoya-u.ac.jp

Naoya Asai's present address is Department of Pathology, Fujita Health University Graduate School of Medicine, Toyoake, Japan

Tetsuro Taki's present address is Department of Pathology and Clinical Laboratories, National Cancer Center Hospital East, Kashiwa, Japan

Yoshinori Hasegawa's present address is National Hospital Organization Nagoya Medical Center, Nagoya, Japan

Masahide Takahashi's present address is International Center for Cell and Gene Therapy, Fujita Health University, Toyoake, Japan 
have consistently indicated that TGF- $\beta$ signaling in CAFs is correlated with immune evasion and immunotherapy failure (Chakravarthy et al, 2018; Mariathasan et al, 2018). However, a complete picture of the roles of diverse CAFs in tumor immunity and responses to ICB is still lacking. It is also unclear whether a specific CAF subset enhances the efficacy of ICB therapy.

We recently described a novel CAF subset characterized by the expression of Meflin (also known as ISLR), a glycosylphosphatidylinositol (GPI)-anchored membrane protein, in pancreatic and colorectal cancers (Mizutani et al, 2019; Kobayashi et al, 2021; Takahashi et al, 2021; Ichihara et al, 2022). Histological and single-cell analyses demonstrated that Meflin-positive (Meflin ${ }^{+}$) CAFs are weakly positive or negative for $\alpha$-SMA mRNA and are distinct from conventional strongly $\alpha$-SMA-positive CAFs (Mizutani et al, 2019). Analyses of mouse tumor models and human tissue samples suggested that the function of Meflin ${ }^{+}$CAFs is the suppression, and not progression, of cancer (Mizutani et al, 2019; Kobayashi et al, 2021). Biochemical analyses showed that Meflin binds to bone morphogenetic protein 7 (BMP7) to augment its signaling, which is known to inhibit the activity of TGF- $\beta$. This suggests that Meflin suppresses various TGF- $\beta$-induced responses, such as tissue fibrosis (Hara et al, 2019; Nakahara et al, 2021). Based on these findings, we propose that Meflin is a specific marker of tumorrestraining CAFs (rCAFs), the existence of which has been postulated previously (Lee et al, 2014; Rhim et al, 2014; Shin et al, 2014; Özdemir et al, 2014). However, the role of Meflin ${ }^{+}$CAFs in the tumor response to ICB therapy remains unclear.

In the present study, we showed that the proportion of Meflin ${ }^{+}$ CAFs in tumor stroma correlated with a favorable response to ICB therapy in patients with non-small cell lung cancer (NSCLC) and mouse syngeneic tumor models. NSCLC tumors with a high number of Meflin ${ }^{+}$CAFs exhibited increased $\mathrm{CD}^{+}{ }^{+}$-cell infiltration and areas of tumor vessels, suggesting the involvement of Meflin ${ }^{+}$CAFs in multiple aspects of the TME. To our knowledge, this is the first study revealing the presence of CAFs that enhance the response to ICB therapy.

\section{Results}

\section{Meflin is a marker of CAFs present in the stroma of invasive NSCLC tumors}

We first examined the expression of Meflin in human lung adenocarcinoma (LUAD) tissues. RNA in situ hybridization (ISH) analysis revealed no apparent Meflin ${ }^{+}$cells in the human lung tissue adjacent to the tumors (Figs $1 \mathrm{~A}$ and S1). In contrast, many Meflin ${ }^{+}$cells appeared in the extensive fibroinflammatory stroma within invasive tumors (INV) (Figs 1A and S1). Interestingly, Meflin ${ }^{+}$stromal cells were not observed in noninvasive tumors (adenocarcinoma in situ; AIS), whereas they were sparsely present in preinvasive lesions (PIL) with a lepidic growth, a pattern of noninvasive cell proliferation along preexisting alveolar wall, adjacent to invasive tumors (Figs $1 \mathrm{~A}$ and S1). Meflin expression was also observed in stromal cells that accumulate in tumors developed in an autochthonous LUAD mouse model (KP mice), harboring K-ras ${ }^{\mathrm{G} 12 \mathrm{D}}$ and p53 null alleles, after the administration of adenovirus-expressing Cre recombinase (DuPage et al, 2009; Taki et al, 2020), whereas it was hardly detected in the normal or tumor adjacent tissue (Fig 1B). Statistical analyses showed that the prevalence of Meflin ${ }^{+}$cells was positively correlated with an increase in the invasiveness of both human and mouse LUAD tumors (Fig 1 C and D). Further fluorescent ISH experiments showed no Meflin expression in E-cadherin ${ }^{+}$epithelial cells, including tumor cells, $\mathrm{CD}_{31}{ }^{+}$endothelial cells, or leukocyte common antigen (LCA) ${ }^{+}$leukocytes (Fig S2A). Meflin expression was observed to a varying degree in cells positive for CAF marker proteins, such as collagen type I $\alpha 1$ (COL1A1), $\alpha$-SMA, and podoplanin (PDPN) (Fig S2B). These data showed that Meflin is a marker of CAFs that accumulate in the invasive stages of both human and mouse LUAD. CAF-specific expression of Meflin was also confirmed by the analysis of single-cell RNA sequencing data of whole cells isolated from human NSCLC and distal non-malignant lung samples (ArrayExpress accession numbers E-MTAB-6149 and E-MTAB-6653, Lambrechts et al, 2018) (Fig 1E).

We observed that CAFs accumulating in human NSCLC exhibited variable expression of Meflin and other CAF markers at the mRNA level (Figs 1F and S2C and D). Duplex ISH staining showed that a substantial fraction of CAFs positive for platelet-derived growth factor receptor $\alpha$ (PDGFR $\alpha$ ), an established fibroblast marker, was also positive for Meflin. In contrast, the expression of $\alpha$-SMA, a marker of myCAFs (Öhlund et al, 2017; Elyada et al, 2019), was inversely correlated with Meflin expression; 12\% of CAFs expressing $\alpha$-SMA were positive for Meflin, indicating that CAFs with high Meflin expression exhibited low or negative $\alpha$-SMA expression (Figs $1 F$ and S2C and D). Meflin was expressed in $~ 33 \%$ and $12 \%$ of FAP $^{+}$and $\mathrm{PDPN}^{+}$CAFs, respectively. Further analysis focusing on the fibroblast cluster of the single-cell RNA sequencing data (Lambrechts et al, 2018) confirmed that Meflin expression was enriched in ACTA2 ${ }^{\text {low/neg }}, I L 6^{\text {low } / \text { neg }}$, or HLA-DRA low/neg subsets, indicating that Meflin $^{+}$CAFs represent a CAF subset distinct from myCAF, iCAF, and apCAF (Fig 2). These observations suggest that Meflin is a marker of $\mathrm{PDGFR}^{+/-} \alpha$-SMA ${ }^{\text {low/neg }} \mathrm{FAP}^{+/-}$PDPN ${ }^{\text {low/neg }}$ CAFs in human NSCLC.

\section{Heterogeneous expression of Meflin in CAFs among patients with NSCLC}

Given that previous studies have shown that the expression of Meflin, an rCAF marker, is heterogeneous among patients with pancreatic and colorectal cancers (Mizutani et al, 2019; Kobayashi et al, 2021), we evaluated the prevalence of Meflin ${ }^{+}$CAFs in patients with NSCLC who did not receive ICB treatment. To this end, we investigated Meflin expression by ISH in NSCLC samples surgically resected at our institution. As observed in LUAD samples, Meflin expression was explicitly observed in CAFs in the stroma of lung squamous cell carcinoma (LUSC) tissues (Fig 3A). To quantify Meflin ${ }^{+}$ CAFs, we assigned all stromal cells with oval- to spindle-shaped nuclei as CAFs, excluding lymphocytes, erythrocytes, endothelial cells, and macrophages, based on their morphologies revealed by hematoxylin counterstain. Then, we semiquantitatively scored the expression of Meflin in each patient according to the percentage of Meflin ${ }^{+}$CAFs in total CAFs as described in the Materials and Methods section. Interestingly, the analysis revealed that patients with NSCLC showed a two-peak distribution with Meflin scores at the peaks of 5 and 25, respectively (Fig 3B). After the data and the criterion described previously (Mizutani et al, 2019), we set the threshold of Meflin-high as 
A Tumor adjacent Adenocarcinoma Preinvasive lesion Invasive adeno-
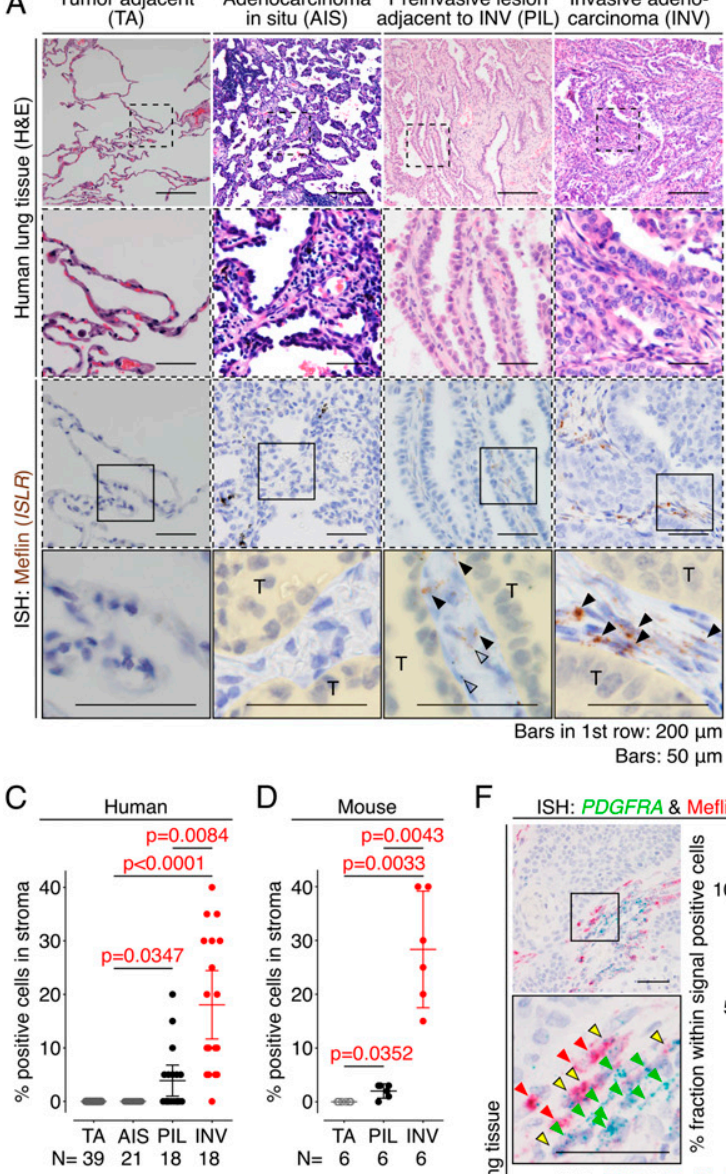

ISH: PDGFRA\&
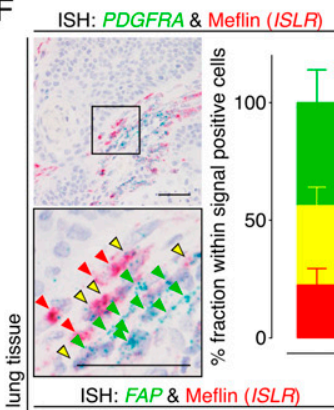

E

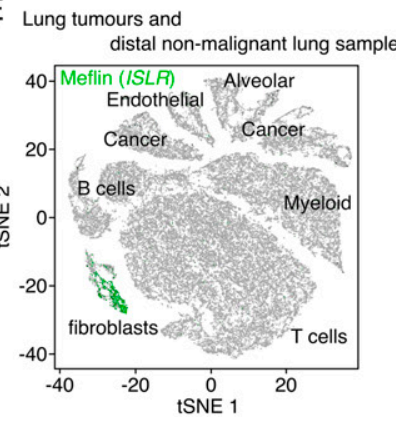

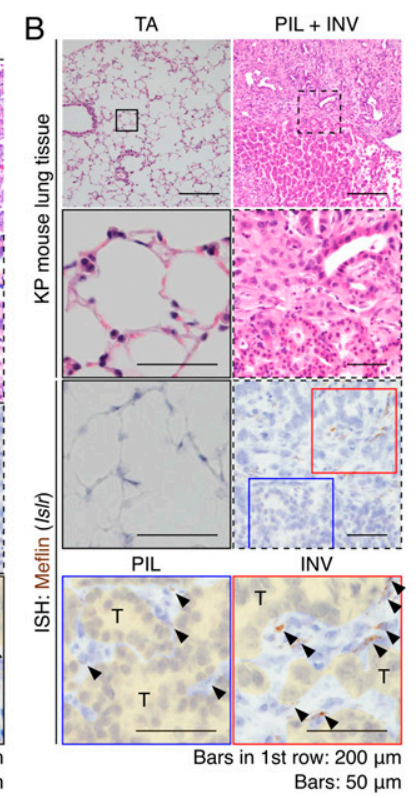

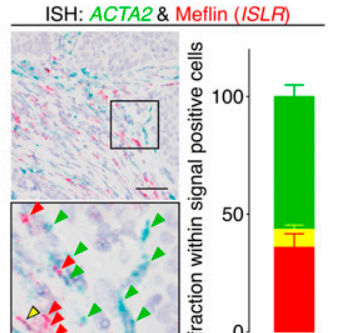

Figure 1. Meflin is a marker of cancer-associated fibroblasts (CAFs) that appear in invasive human non-small cell lung cancer (NSCLC).

(A) Tissue sections derived from noninvasive (AIS) and invasive (INV) LUAD tumors were stained for H\&E (top panels) and Meflin mRNA by ISH (lower panels). Preinvasive lesions adjacent to the invasive tumor (PIL) and tumor adjacent tissue (TA) were also examined. Boxed areas were magnified in lower panels. Black and open arrowheads denote Meflin+ stromal cells and Meflin- macrophages that phagocytize foreign material, respectively. Areas filled with yellow indicate tumor parenchyma comprised of tumor cells $(T)$. (B) Preinvasive (PIL) and invasive (INV) lesions of a tumor developed in the autochthonous LUAD mouse model (KP mice) were examined for Meflin expression by ISH. Meflin+ stromal cells (arrowheads) were found in the INV and PIL lesion but not in tumor adjacent tissue (TA). (C, D) Quantification of the percentage of Meflin ${ }^{+}$cells in all cells with oval- to spindle-shaped nuclei found in the stroma of the tumor adjacent (TA), noninvasive (AIS), preinvasive (PIL), and invasive (INV) cases and area of human LUAD cases (C) and the KP mouse model (D), respectively. All stromal cells found in high-power fields (400x) of the indicated number of cases were evaluated.

(E) Analysis of a single-cell RNA sequencing dataset showed the specific expression of Meflin in fibroblasts in human NSCLC and distal non-malignant lung samples. (F) Duplex ISH for Meflin (red) and other CAF markers (green) showed that Meflin is variably coexpressed with other established CAF markers (PDGFRA, ACTA2, FAP, or PDPN) in CAFs of human NSCLC. Green, yellow, and red arrowheads denote cells that are single-positive for the indicated CAF markers, double-positive for the indicated CAF markers and Meflin, and single-positive for Meflin, respectively. All stromal cells that were positive for ISH signals in high-power fields from three independent NSCLC tumors were evaluated and quantified. The graphs show the percent fraction of each subset within ISH signal positive cells.

Source data are available for this figure.
$20 \%$ or more fibroblasts expressing Meflin, and we used this criterion in the experiment shown below (Fig 3B).

We also confirmed that Meflin expression in CAFs in biopsy samples could be evaluated by ISH (Fig S3). However, we found that some biopsy samples did not contain any stromal components, which made it difficult to examine Meflin expression (Fig S3). We considered these subjects as inappropriate samples and excluded them from the study shown below.

\section{High infiltration of Meflin ${ }^{+}$CAFs correlates with favorable response to ICB in patients with NSCLC}

Next, we investigated the involvement of Meflin ${ }^{+}$CAFs in tumor response to ICB therapy. We conducted a retrospective observational study of 132 patients with NSCLC who had received ICB monotherapy targeting programmed cell death 1 (PD-1) (nivolumab or pembrolizumab) or programmed cell death 1 ligand 1 (PD-L1) (atezolizumab) at our institution (Fig 4A). The patients were divided into Meflin-high ( $220 \%$ Meflin $^{+}$CAFs) and Meflin-low ( $<20 \%$ Meflin $^{+}$CAFs) groups by ISH analysis (Fig 4B) following the criterion described above. We also evaluated the average total numbers of fibroblasts based on cell morphology, and found that they were comparable between the Meflin-high and Meflin-low groups (Fig 4C). A total of 98 patients were analyzed for outcomes, including objective response rate (ORR) assessed by immunotherapy Response Evaluation Criteria in Solid Tumors (iRECIST) (Seymour et al, 2017), overall survival (OS), and progression-free survival (PFS) (Fig 4A and Table 1). The exclusion criteria are described in Fig $4 \mathrm{~A}$. 

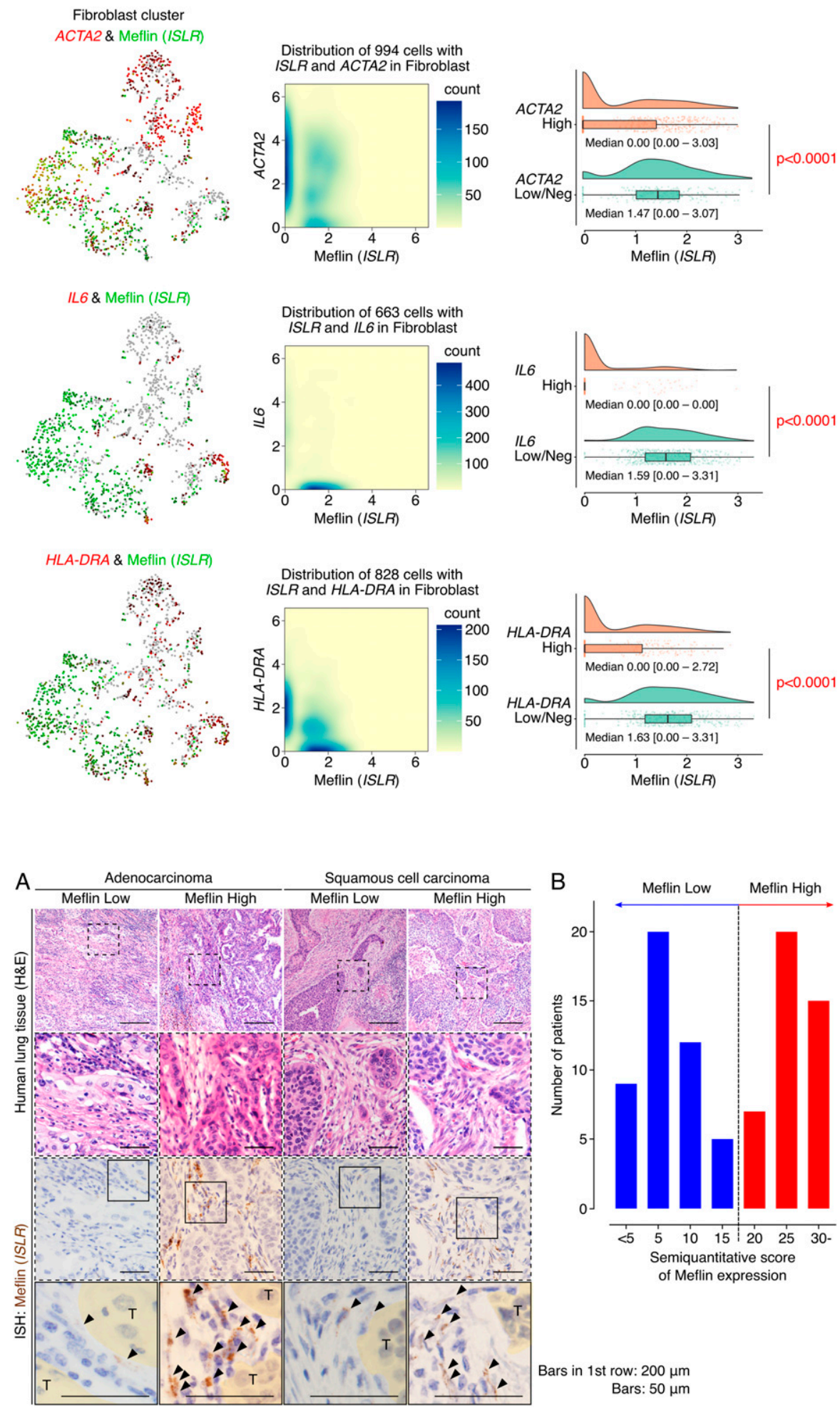

Figure 2. Meflin $^{+}$cancer-associated fibroblasts (CAFs) constitute a CAF subset distinct from known CAF subsets.

t-distributed stochastic neighbor embedding (tSNE) plots obtained by analysis of a single-cell RNA sequencing dataset showed differential expression of Meflin in $\mathrm{ACTA2}^{+}, 1 \mathrm{L6} 6^{+}$and HLA-DRA ${ }^{+}$CAF subsets, which represent myCAFs, iCAFs, and apCAFs, respectively, of human non-small cell lung cancer and distal nonmalignant lung samples (left panels). Density plots (middle panels) and violin/box/scatter plots (right panels) derived from gene expression data of the single-cell analysis showed that Meflin ${ }^{+}$CAFs constitute a unique CAF subset distinct from myCAF, iCAFs, and apCAFs.

Source data are available for this figure.
Figure 3. Meflin expression in cancer-associated fibroblasts (CAFs) is heterogeneous and shows a two-peak distribution across patients with non-small cell lung cancer.

(A) Representative images of Meflin-high and Meflinlow cases of invasive adenocarcinoma (left) and squamous cell carcinoma (right). Dashed boxed areas were magnified in middle panels, which showed serial sections stained for H\&E and Meflin mRNA by ISH. In the images of Meflin ISH, boxed areas were magnified in the lowest panels. Areas filled with yellow denote tumor cells $(T)$. Arrowheads indicate Meflin ${ }^{+}$CAFs. (B) Distribution of patients with non-small cell lung cancer stratified by the percentage of Meflin ${ }^{+}$CAFs in all stromal cells. The number of Meflin ${ }^{+}$ CAFs was counted in randomly selected five high-power microscopic fields. The proportion of Meflin ${ }^{+}$CAFs was represented as the ratio of Meflin ${ }^{+}$CAFs to all stromal cells with oval to spindle-shaped nuclei. Meflinhigh was defined as $\geq 20 \%$ of stromal cells positive for Meflin.

Source data are available for this figure.
Interestingly, the data showed that ORR of the Meflin-high group (40.3\%, 25 of 62 patients) was significantly higher than that of the Meflin-low group ( $0 \%, 0$ of 32 patients) $(P<0.0001$, Fig 4D). The threshold of $20 \%$ Meflin positivity in all CAFs was found to be the best criterion for predicting response to ICB monotherapy, with an area under the receiver operating characteristic curve (AUC) of 0.632 (95\% Cl, 0.526-0.738) (Fig 4E). Kaplan-Meier survival analyses using the log-rank Mantel-Cox test revealed that the Meflin-high group had a significantly favorable prognosis in both OS $(P=0.0281)$ and PFS $(P=0.0011)$ than the Meflin-low group (Fig 4F). The analysis 
A
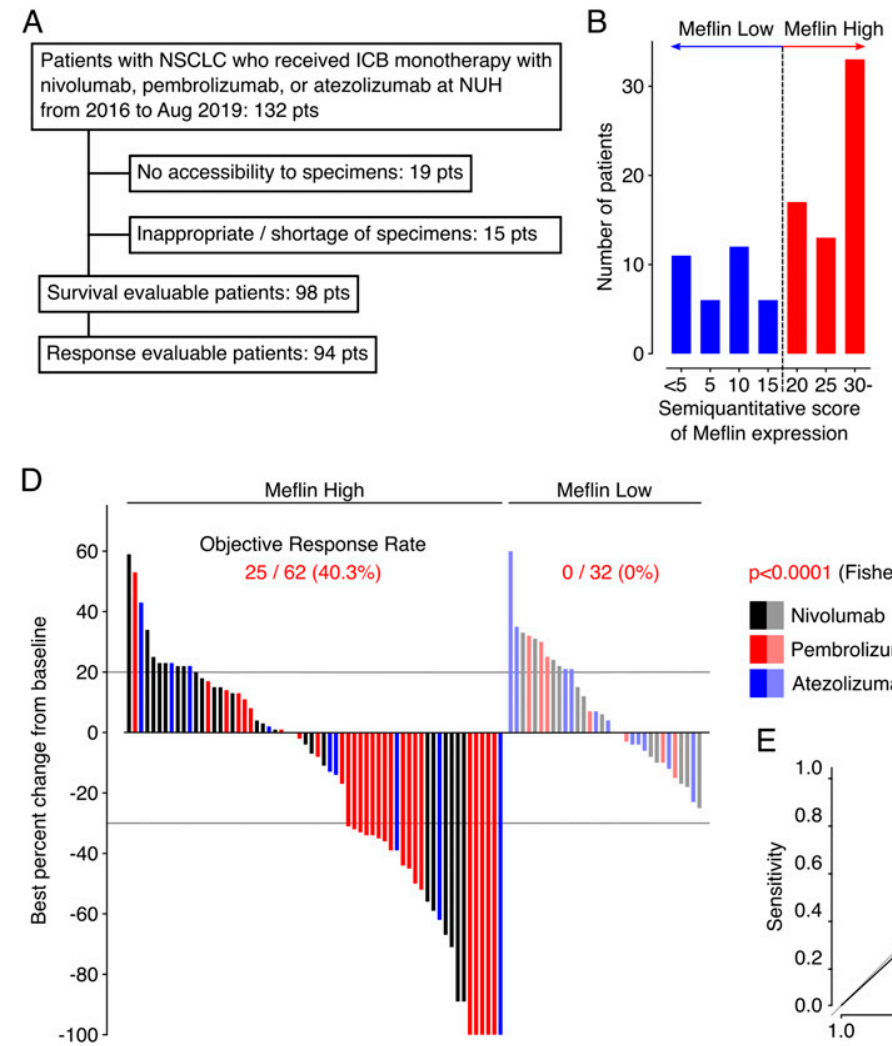

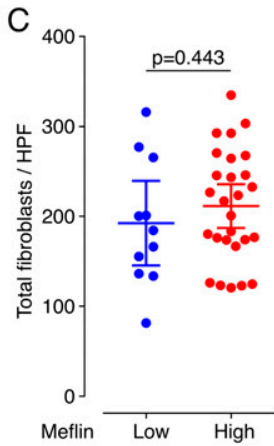

Figure 4. Non-small cell lung cancer (NSCLC) patients with high Meflin ${ }^{+}$cancer-associated fibroblast (CAF) infiltration exhibit favorable responses to immune checkpoint blockade (ICB) therapy.

(A) Flow diagram of the selection of eligible patients with NSCLC who received ICB monotherapy for objective response rate analysis in our institution. (B) Distribution of patients with NSCLC stratified by the proportion of Meflin ${ }^{+}$CAFs determined by ISH analysis. The number of Meflin ${ }^{+}$CAFs was counted in randomly selected four high-power microscopic fields. The proportion of Meflin ${ }^{+}$CAFs was calculated as the ratio of Meflin ${ }^{+}$cells to all stromal cells with a spindle morphology. Meflin-high was defined as $\geq 20 \%$ of stromal cells stained for Meflin by ISH. (c) Total numbers of fibroblasts found in tissue sections obtained from Meflin-high and -low groups were counted and quantified. NSCLC cases whose surgical specimens were available $(n=38)$ were evaluated. HPF, high-power field. (D) A waterfall plot showing changes in tumor size from baseline determined according to iRECIST criteria in Meflin-high (left) and Meflin-low (right) patients with NSCLC who receive ICB monotherapy. Black, red, and blue bars indicate patients treated with nivolumab, pembrolizumab, and atezolizumab, respectively. (E) A receiver operating characteristics (ROC) curve for the percentage of Meflin ${ }^{+}$CAFs in tumor stroma. The ROC curve was obtained by plotting sensitivity against specificity at each threshold setting. The area under the curve (AUC) (0.632; 95\% Cl, 0.526-0.738) shown in the plot summarizes the performance of Meflin ${ }^{+}$CAFs in tumor stroma. (F) OS (left) and progression-free survival (right) of Meflin-high (red) and Meflin-low (blue) NSCLC patients treated with ICB therapy. The Meflin-high group showed a favorable response to ICB therapy compared with the Meflin-low group. Shown in the boxes below the plots are the observed numbers of events (deaths or disease progression) and median survival (months) of Meflin-high and Meflin-low groups over the follow-up periods. Mo, months.

Source data are available for this figure.

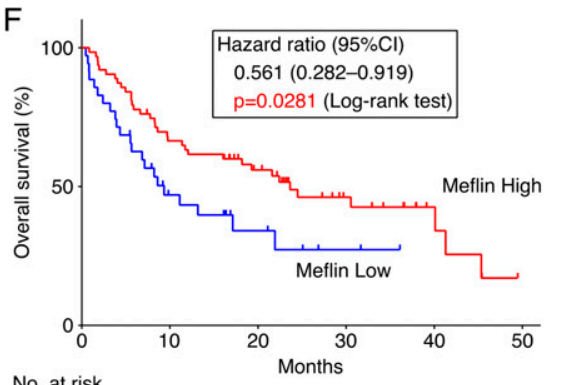

\begin{tabular}{|c|c|c|c|c|c|c|}
\hline \multicolumn{7}{|c|}{ o. at risk } \\
\hline High & 63 & 41 & 27 & 13 & 5 & 0 \\
\hline Low & 35 & 13 & 6 & 2 & 0 & 0 \\
\hline \multicolumn{3}{|c|}{ Meflin } & \multicolumn{2}{|r|}{ High } & \multicolumn{2}{|c|}{ Low } \\
\hline \multirow{2}{*}{\multicolumn{3}{|c|}{ No. of events (\%) }} & \multicolumn{2}{|c|}{$35(55.6 \%)$} & \multicolumn{2}{|c|}{$22(62.9 \%)$} \\
\hline \multicolumn{2}{|c|}{ Mo, median $(95 \% \mathrm{Cl})$} & & 23. & (12.1-41.3) & 9.34 & $52-21.9)$ \\
\hline
\end{tabular}

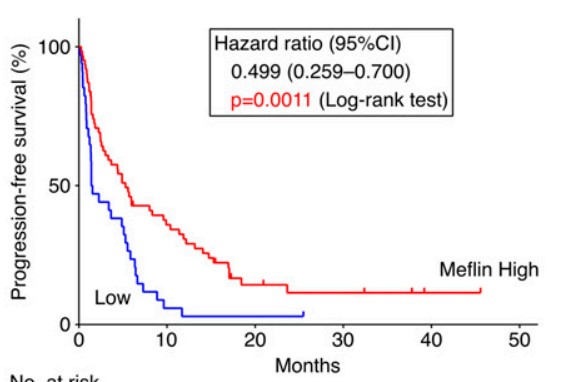

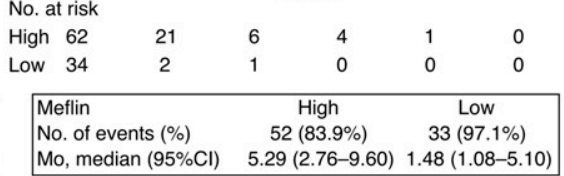

of the data using a multivariate Cox proportional hazard regression model showed a positive correlation between the percentage of Meflin ${ }^{+}$CAFs and the outcomes (Table 2). Consistent with recent studies that showed that PD-L1 tumor proportion score (TPS) does not necessarily predict the response to ICB therapy (Carbone et al, 2017; Shen \& Zhao, 2018; Lu et al, 2019), the analysis of PD-L1 expression in tumor cells, which was obtained with the 22C3 clone on the Dako Autostainer Link 48 platform, showed that PD-L1 TPS did not correlate with ORR, OS, or PFS in our NSCLC cohort (TPS < 1\%; 22.7\% ORR, TPS 1-49\%; 14.3\%, and TPS $\leq 50 \%$; 38.2\%) ( $P=0.071$, Fig S4A-C and Table 2). These data demonstrate that Meflin expression in CAFs is a predictive marker for the response to ICB in patients with NSCLC.

\section{Meflin expression in CAFs correlates with high infiltration of $\mathrm{CD}^{+}$ $T$ cells and tumor vessel area}

We next examined the correlation between Meflin expression in CAFs and the profiles of tumor-infiltrating lymphocytes (TILS) using an automated imaging system and a user-trainable image analysis software as described in the Materials and Methods section. Seven-color multiplex immunofluorescence (IF) staining of the specimens of 32 surgically resected NSCLC tumors who received ICB monotherapy revealed that the number of $\mathrm{CD}^{+} \mathrm{T}$ cells infiltrating the stroma (interstitium), but not the tumor parenchyma, was significantly higher in Meflin-high patients than in Meflin-low patients (Fig 5A). In contrast, the numbers of $\mathrm{CD}^{+} \mathrm{T}$ cells and 


\section{Life Science Alliance}

Table 1. Characteristics of patients who received immune checkpoint blockade monotherapies.

\begin{tabular}{|c|c|c|c|}
\hline \multirow[b]{2}{*}{ Variable } & \multicolumn{2}{|c|}{ Meflin expression } & \multirow{2}{*}{$P$-value } \\
\hline & High & Low & \\
\hline Median age-yr (range) & $70(42-85)$ & $69(43-80)$ & $0.920^{\mathrm{b}}$ \\
\hline Sex-no. (\%) & & & 0.663 \\
\hline Female & $23(36.5 \%)$ & $11(31.4 \%)$ & \\
\hline Male & $40(63.5 \%)$ & $24(68.6 \%)$ & \\
\hline Subtype-no. (\%) & & & 0.0813 \\
\hline Squamous cell carcinoma (LUSC) & $17(27.0 \%)$ & $3(8.6 \%)$ & \\
\hline Adenocarcinoma (LUAD) & $37(58.7 \%)$ & $27(77.1 \%)^{c}$ & \\
\hline Others & $9(14.3 \%)$ & $5(14.3 \%)$ & \\
\hline Targetable EGFR mutation in LUAD-no. (\%) & & & $>0.999$ \\
\hline Not detected & $26(70.3 \%)$ & $17(68.0 \%)^{c}$ & \\
\hline Detected & $11(29.7 \%)$ & $8(32.0 \%)$ & \\
\hline PD-L1 TPS ${ }^{d}$-no. (\%) & & & 0.139 \\
\hline$<1 \%$ & $16(26.2 \%)$ & $7(20.6 \%)$ & \\
\hline $1-49 \%$ & $18(29.5 \%)$ & $17(50.0 \%)$ & \\
\hline $50 \% \leq$ & $27(44.3 \%)$ & $10(29.4 \%)$ & \\
\hline Brinkman index - no. (\%) & & & 0.525 \\
\hline$<400$ & $26(41.3 \%)$ & $12(34.3 \%)$ & \\
\hline $400 \leq$ & 37 (58.7\%) & $23(65.7 \%)$ & \\
\hline ECOG-PS-no. (\%) & & & 0.255 \\
\hline $2 \leq$ (Poor) & $8(12.7 \%)$ & $8(22.9 \%)$ & \\
\hline 0 or 1 (Good) & $55(87.3 \%)$ & $27(77.1 \%)$ & \\
\hline Tx line ${ }^{f}-$ no. (\%) & & & 0.0046 \\
\hline $1 \mathrm{~L}$ or $2 \mathrm{~L}$ & $46(73.0 \%)$ & $15(42.9 \%)$ & \\
\hline $3 \mathrm{~L} \leq$ & $17(27.0 \%)$ & $20(57.1 \%)$ & \\
\hline
\end{tabular}

${ }^{a}$ Fisher's exact test.

${ }^{\mathrm{b}}$ Mann-Whitney U test.

'One patient with anaplastic lymphoma kinase (ALK)-rearranged non-small cell lung cancer

${ }^{\mathrm{d}}$ Tumor Proportion Score was the percentage of viable tumor cells showing partial or complete membrane staining at any intensity.

eBrinkman index was defined as the number of cigarettes smoked per day multiplied by the number of years of smoking (e.g., if one smoked 20 cigarettes per day for $20 \mathrm{yr}$, the Brinkman index is 400).

fOnly conventional cytotoxic chemotherapeutics were considered in the treatment line.

$P$-values in bold showed statistically significant differences.

$\mathrm{CD}^{+}{ }^{+} \mathrm{FoxP} 3^{+}$regulatory $\mathrm{T}$ cells were comparable between the two groups (Fig 5A). There was also no difference in the numbers of $\mathrm{CD} 5 \mathrm{RO}^{+}$memory $\mathrm{CD}^{+} \mathrm{T}$ cells, $\mathrm{CD}^{+} 5 \mathrm{RO}^{+}$memory $\mathrm{CD}^{+} \mathrm{T}$ cells, or $\mathrm{CD}_{20} \mathrm{O}^{+} \mathrm{B}$ cells in both the stroma and tumor parenchyma between the two groups (Fig S5).

Our previous study showed that tumors developed in the pancreas of Meflin KO mice exhibited a decrease in tumor vessel area accompanied by changes in collagen configuration (Mizutani et al, 2019). Higher tumor vascularity is also associated with better tumor responses to ICB therapy in mouse models (Zheng et al, 2018). Immunostaining of the NSCLC tumor samples with anti-CD31 antibody showed that the Meflin-high group tumors had greater tumor vessel area than the Meflin-low group (Fig 5B). These data suggest that the infiltration of Meflin ${ }^{+}$CAFs is associated with increased tumor vessel perfusion.

\section{Defective response of tumors to ICB therapy in Meflin-KO mice}

Next, we determined whether Meflin expression in CAFs is crucial for the response of tumors to ICB using C57BL/6J wild-type (WT) mice and Meflin-KO mice. We previously reported that Meflin-KO mice displayed decreased spleen weight compared with WT mice (Maeda et al, 2016). Therefore, we first examined the immunophenotype of lymphocytes isolated from the spleen of Meflin-KO mice to compare it with that of WT mice. The data showed no differences in the proportions of $\mathrm{CD}^{+}, \mathrm{CD}^{+}$, and regulatory T cells (Fig S6A and B).

Our first attempts on mice bearing tumors of murine Lewis lung carcinoma (LLC) cells were unsuccessful, showing that LLC syngeneic lung tumors developed in C57BL/6) mice were not responsive to either anti-mouse PD-1 (mPD-1) or anti-mouse PD-L1 antibody. We therefore next subcutaneously transplanted syngeneic MC-38 colorectal cancer 
Table 2. Hazard ratios and P-values for multivariate Cox proportional hazard regression model analysis in patients with non-small cell lung cancer subjected to immune checkpoint blockade monotherapies.

\begin{tabular}{|c|c|c|c|c|}
\hline Variable & $\begin{array}{l}\text { Hazard ratio }(95 \% \mathrm{Cl}) \text { for } \\
\text { OS }\end{array}$ & $\begin{array}{l}P \text {-value for } \\
\text { OS }\end{array}$ & $\begin{array}{l}\text { Hazard ratio }(95 \% \mathrm{Cl}) \text { for progression-free } \\
\text { survival }\end{array}$ & $\begin{array}{l}P \text {-value for progression-free } \\
\text { survival }\end{array}$ \\
\hline Age & $0.961(0.934-0.990)$ & 0.0085 & $0.955(0.928-0.983)$ & 0.0018 \\
\hline Subtype & & $0.866^{\mathrm{a}}$ & & $0.849^{a}$ \\
\hline LUSC & Reference & & Reference & \\
\hline LUAD & $0.790(0.334-1.87)$ & 0.592 & $0.771(0.289-2.06)$ & 0.604 \\
\hline Others & $0.847(0.293-2.45)$ & 0.760 & $0.884(0.307-2.55)$ & 0.819 \\
\hline PD-L1 TPS & & $0.128^{a}$ & & $0.919^{a}$ \\
\hline$<1 \%$ & Reference & & Reference & \\
\hline $1-49 \%$ & $0.979(0.441-2.17)$ & 0.958 & $1.06(0.548-2.04)$ & 0.868 \\
\hline $50 \% \leq$ & $1.84(0.858-3.94)$ & 0.117 & $0.927(0.473-1.82)$ & 0.825 \\
\hline \multicolumn{5}{|c|}{$\begin{array}{l}\text { Targetable EGFR } \\
\text { mutation }\end{array}$} \\
\hline Not detected & Not included ${ }^{\mathrm{b}}$ & & Reference & \\
\hline Detected & & & $2.49(1.06-5.87)$ & 0.0374 \\
\hline \multicolumn{5}{|c|}{ Brinkman index } \\
\hline$<400$ & Reference & & Reference & \\
\hline $400 \leq$ & $0.398(0.189-0.836)$ & 0.0151 & $0.706(0.340-1.47)$ & 0.349 \\
\hline \multicolumn{5}{|l|}{ ECOG-PS } \\
\hline $2 \leq$ (Poor) & Reference & & Reference & \\
\hline 0 or 1 (Good) & $0.0468(0.0204-0.108)$ & $<0.0001$ & $0.0994(0.0437-0.226)$ & $<0.0001$ \\
\hline \multicolumn{5}{|l|}{ Tx line } \\
\hline $1 \mathrm{~L}$ or $2 \mathrm{~L}$ & Reference & & Reference & \\
\hline $3 \mathrm{~L} \leq$ & $1.12(0.604-2.09)$ & 0.712 & $0.803(0.450-1.43)$ & 0.458 \\
\hline \multicolumn{5}{|l|}{ Meflin } \\
\hline Low & Reference & & Reference & \\
\hline High & $0.473(0.242-0.922)$ & 0.0280 & $0.486(0.268-0.881)$ & 0.0174 \\
\hline
\end{tabular}

awald test.

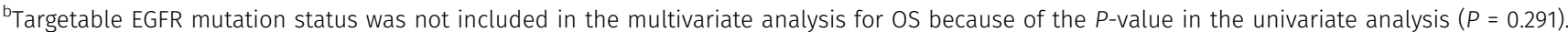

$P$-values in bold showed statistically significant differences.

(CRC) cells, a well-established cell line that is used to study the antitumor effect of ICB therapy (House et al, 2020; Mager et al, 2020), into WT mice and Meflin-KO mice, followed by intraperitoneal administration of anti-mPD-1 antibody or isotype control IgG on day 4, 7, and 10 after transplantation (Fig 6A). WT mice treated with anti-mPD-1 antibody, but not isotype control IgG, had a statistically better prognosis than Meflin-KO mice (Fig 6B). The effect of Meflin deficiency on the antitumor effect of mPD-1 antibody was also evaluated using a linear mixed-effect model with restricted maximum likelihood estimates, which showed that the suppressive effect of anti-mPD-1 antibody on tumor growth was significantly weakened by Meflin-KO $(P=$ 0.0041), although Meflin-KO itself did not exhibit altered tumor growth $(P=0.901$, Fig 6C).

The role of Meflin in promoting the antitumor effect of anti-mPD-1 antibody was also confirmed in another experimental setup, in which we orthotopically transplanted syngeneic EO771 breast cancer (BC) cells (House et al, 2020; Zheng et al, 2018) into the fourth right mammary fat pad of WT mice and Meflin-KO mice, followed by intraperitoneal administration of anti-mPD-1 antibody or control IgG on day 6, 9, and 12 after the transplantation (Fig 6D). On day 19, a suppressive effect of anti-mPD-1 antibody on tumor volumes was observed, which was abrogated in Meflin-KO mice as indicated by the P-value of 0.0227 obtained according to the two-sided permutation Brunner-Munzel test with Holm-Bonferroni correction (Fig 6E). The importance of Meflin expression in CAFs upon anti-mPD-1 antibody treatment was measured by the effect size (Cliff's delta) of -0.796 ( $95 \% \mathrm{Cl}-1.00$ to -0.184 ) (Fig 6E). Consistent with the analysis of human NSCLC samples, the tumor vessel area in EO771 tumors developed in WT mice was greater than that in Meflin-KO mice (Fig 6F). Taken together, Meflin expression in CAFs might facilitate the antitumor effect of anti-mPD-1 antibody by increasing the tumor vascular bed.

\section{Meflin expression in CAFs associates with TIL activation in mice}

We then explored the status of TILS in MC-38 tumors developed in WT and Meflin-KO mice (Fig 7A). Unfortunately, the infiltration of 


\section{A}
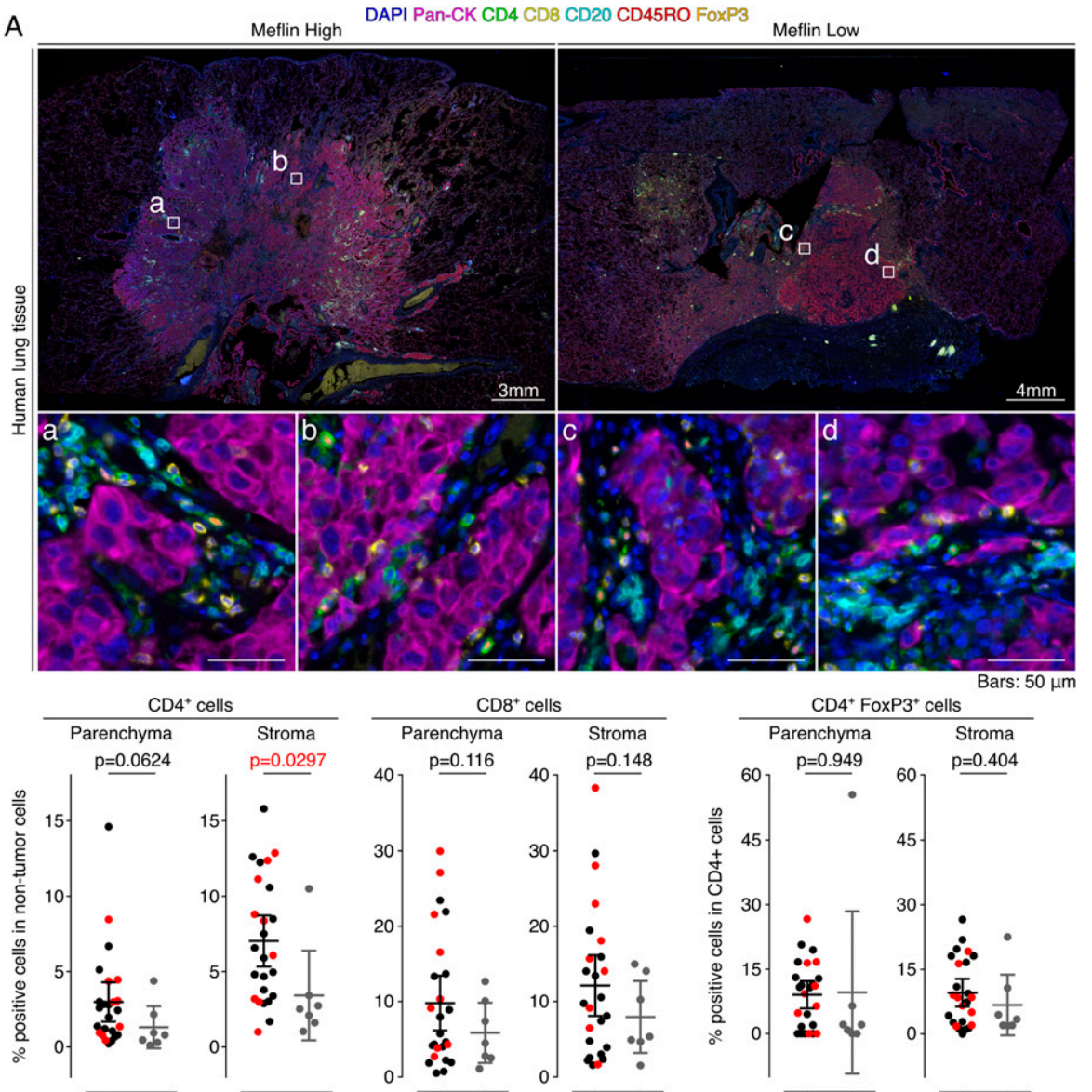

Meflin High Low

B

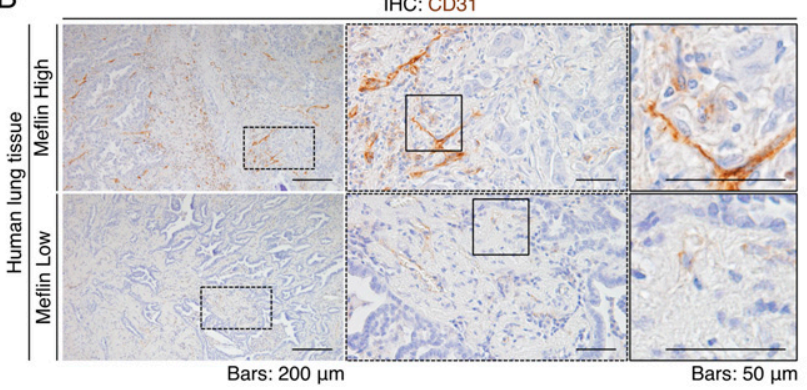

Figure 5. Meflin expression in cancer-associated fibroblasts correlates with tumor infiltration of $\mathrm{CD}^{+} \mathrm{T}$ cells and vascular area in non-small cell lung cancer patients treated with immune checkpoint blockade (ICB) monotherapy.

(A) Tissue sections prepared from tumors of patients with non-small cell lung cancer who received ICB monotherapy were stained by multiplex immunofluorescence for the indicated TIL markers, nuclei, and pan-cytokeratin (Pan-CK), followed by imaging with a multispectral imaging system.

Representative images of Meflin-high (left, $n=25$ ) and Meflin-low (right, $n=7$ ) cases are shown. Boxed regions were magnified in lower panels. The lower panels' graphs show the quantification of the percentage of $\mathrm{CD}^{+}, \mathrm{CD}^{+}$, and $\mathrm{CD}_{4}{ }^{+} \mathrm{FoxP}^{+} \mathrm{T}$ cells in all TILs infiltrated in intra-tumor (parenchyma) and stroma regions of each group. Red dots in the graphs denote the responders to ICB monotherapy. (B) Representative images of tissue sections of Meflin-high (upper panel) and Meflin-low (lower panel) tumors stained for CD31. The graph on the right shows the quantification of tumor vessel areas in each group. High magnification views randomly selected from 25 Meflin-high and 7 Meflin-low cases were analyzed and quantified. Red dots in the graphs denote the responders to ICB monotherapy.

Source data are available for this figure.
$\mathrm{CD}^{+} \mathrm{T}$ cells, $\mathrm{CD} 8^{+} \mathrm{T}$ cells, and $\mathrm{CD} 25^{+} \mathrm{FoxP}^{+}$regulatory $\mathrm{T}$ cells varied across two independent experiments. Therefore, we concluded that, contrary to the analysis of human NSCLC tissues, T-cell infiltration was similar between tumors developed in WT and Meflin$\mathrm{KO}$ mice (Fig 7B). Interestingly, we found that the expression of immune checkpoint molecules in some subsets of TILs was higher in tumors of WT mice than that of Meflin-KO mice, which included T-cell immunoglobulin and mucin-domain-containing molecule 3 (TIM-3) on $\mathrm{CD}^{+}$and regulatory $\mathrm{T}$ cells, PD-1, CD25, cytotoxic T-lymphocyte associated protein 4 (CTLA-4), and inducible T-cell co-stimulator (ICOS) on $\mathrm{CD}^{+} \mathrm{T}$ cells (Fig 7C-E). Previous studies have indicated that several molecules such as PD-1 and ICOS on $\mathrm{CD} 8^{+} \mathrm{T}$ cells are associated with the activation of antitumor immunity and favorable clinical responses to ICB therapy (Gros et al,
2014, 2016; Xiao et al, 2020; Kumagai et al, 2020b). These exploratory analyses suggest that Meflin expression in CAFs is associated with TIL activation in mice, but not their recruitment or infiltration into tumors.

\section{Induced expression of Meflin in the lineage of Meflin ${ }^{+}$cells} enhance the antitumor activity of anti-mPD-1 antibody therapy

The findings described above suggested that the induction of Meflin expression in CAFs can be a therapeutic strategy to enhance ICB therapy efficacy. To prove this, we generated a transgenic mouse line expressing mouse Meflin under the tetracycline response element (TRE) promoter (TRE-Meflin) (Fig 8A). This line was then crossed with Meflin-Cre (Hara et al, 2019, 2021; Mizutani et al, 
A
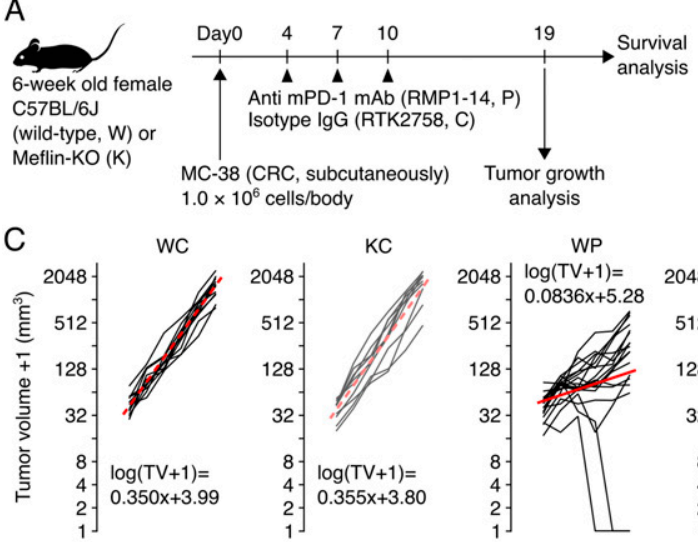

04710
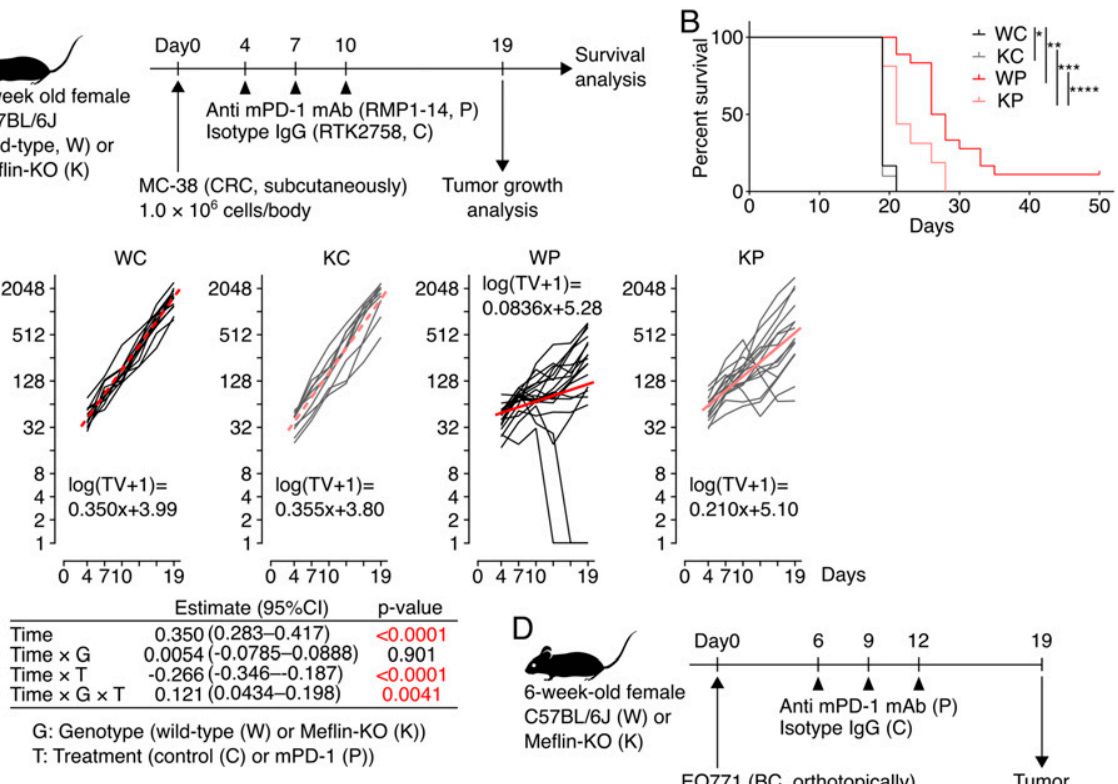

KP
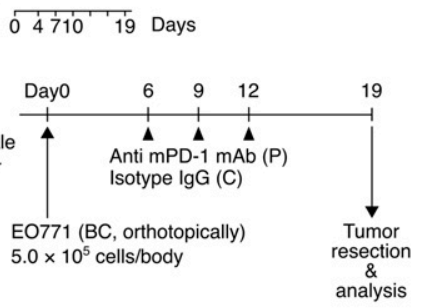

E
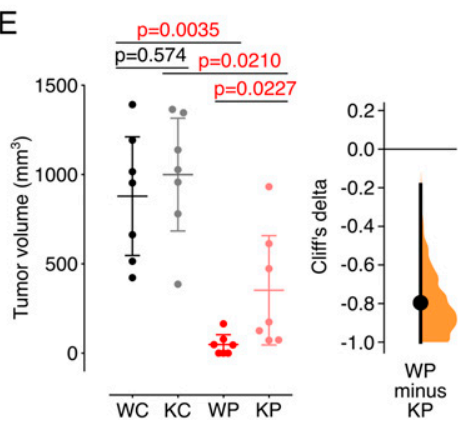

$\mathrm{F}$

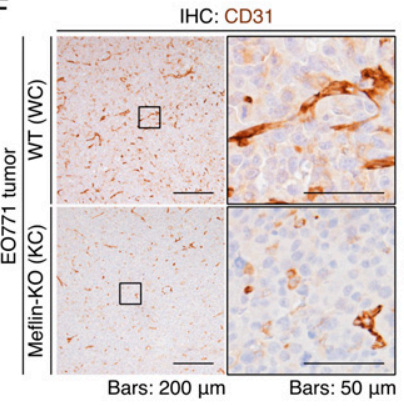

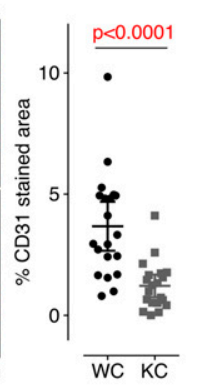

Figure 6. Tumors developed in Meflin-KO mice exhibit poor response to anti-mPD-1 therapy.

(A) An experimental protocol to test the antitumor effect of anti-mPD-1 therapy in mice. MC-38 mouse CRC cells were transplanted into C57BL/ 6 wild-type (W) or Meflin-KO (K) mice at day 0 , followed by the intraperitoneal administration of anti-mPD-1 (P) or an isotype control antibody (C). (B) Survival of wild-type (W) and Meflin-KO (K) mice treated with isotype control (WC and KC, respectively) or treated with anti-mPD-1 antibody (WP and $\mathrm{KP}$, respectively). The numbers of mice tested for $W C, K C, W P$, and KP groups were 12, 10, 18 , and 16 , respectively. ${ }^{*} P=0.658 ;{ }^{* *} P<0.0001$; ${ }^{* * *} P=0.0033 ;{ }^{* * \star} P=0.0033$. (c) Time courses of the volumes of tumors developed in the indicated groups (black and grey lines). For the log transformation of tumor volumes, one was added to every tumor volume. Red lines indicate linear approximations. The table shown under the graphs shows the restricted maximum likelihood estimates of each parameter in a linear mixed-effects model that includes the interactions of time, time and mouse genetic background (G), time and anti-mPD-1 therapy ( $\mathrm{T}$ ), and time and $G$ and $T$ while adding variable effects to the slope and intercept for each individual. (D, E) EO771 mouse $B C$ cells were transplanted into wild-type (W) or Meflin-KO (K) mice at day 0 , followed by the intraperitoneal administration of anti-mPD-1 antibody (P) or an isotype control antibody (C). The number of mice tested for each group was seven. Shown in (E) are the quantification of tumor volume of each group (left) and the nonparametric estimate of effect size calculated by Cliff's delta (right). (F) Tissue sections from EO771 tumors developed in wild-type (WC) and Meflin-KO (KC) were stained for CD31 to visualize tumor vessels (left), followed by the quantification of the stained areas (right). Boxed regions were magnified in adjacent panels.

Source data are available for this figure.
2019) and Rosa26-LSL (LoxP-stop-LoxP)-rtTA3 (third-generation reverse tetracycline-regulated transactivator) mice (JAX stock \#029617, Dow et al, 2014) to generate mice with doxycycline-induced expression of Meflin in Meflin-lineage cells. To confirm induced Meflin expression in mice carrying all three alleles (hereafter referred to as Meflin-TO), we administered doxycycline in drinking water $(2 \mathrm{mg} / \mathrm{ml}$ ) to Meflin-TO mice and transplanted MC-38 and EO771 cells subcutaneously and orthotopically, respectively, followed by the analysis of Meflin expression (Fig S7A). Quantitative PCR (qPCR) of the tumor tissue samples revealed that doxycycline induced Meflin expression in Col1a1 ${ }^{+}$stromal cells, representing CAFs, in tumors developed in Meflin-TO mice administered doxycycline, but not in control mice that lack the Meflin-Cre allele (Fig S7B). ISH and qPCR also confirmed the induced Meflin expression in cultured CAFs isolated from tumors developed in Meflin-TO but not that of control mice (Fig S7C).

Consistent with the analysis of tumor vessel area of human NSCLC tissues and tumors developed in WT and Meflin-KO mice (Figs 5B and 6F), the area of vasculature in tumors developed in doxycycline administered-Meflin-TO was significantly larger than that in Meflin-TO mice not administered doxycycline and control mice that lacked the Meflin-Cre allele (Fig S7D).

Finally, littermates obtained by crossing Meflin-Cre, TRE-Meflin, and Rosa26-LSL-rtTA3 mice were administered doxycycline and subcutaneously transplanted with MC-38 cells, followed by intraperitoneal administration of anti-mPD-1 antibody on day 4, 7, and 10. Genotyping of the mice was performed on day 19 (Fig 8B). The genotypes of the mice were blinded to the investigators during data acquisition and analysis. The results showed that Meflin-TO administered doxycycline and anti-mPD-1 antibody exhibited a more favorable prognosis and response than control mice (Fig $8 \mathrm{C}$ and D). These data supported the notion that Meflin is a CAF marker and functionally contributes to a subset of CAFs that facilitate the antitumor effect of ICB therapy (Fig 8E).

\section{Discussion}

In the present study, we focused on the role of Meflin, a recently identified rCAF marker in pancreatic and colorectal cancers (Mizutani et al, 2019; Kobayashi et al, 2021), in tumor response to ICB therapy through the analyses of human NSCLC samples and syngeneic tumor mouse models. Our data suggest that Meflin expression in CAFs correlates with favorable tumor response to ICB therapy, leading to the hypothesis that Meflin ${ }^{+}$CAFs promote the host antitumor immune response. Previous studies have shown that immunosuppressive CAFs, such as $\alpha-\mathrm{SMA}^{+} \mathrm{FAP}^{+}$CAFs, $\mathrm{LRRC}^{+} 5^{+} \mathrm{CAFs}$, 

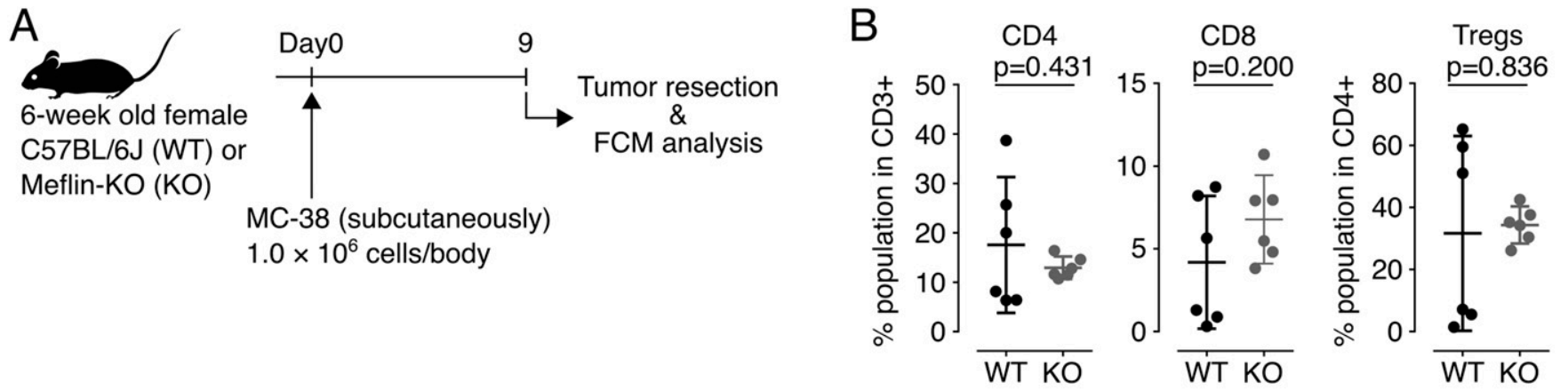

C

$\mathrm{CD}^{+}$cells $\left(\mathrm{CD}^{+}{ }^{+} \mathrm{CD}^{-} 5^{-}\right.$FoxP3 $3^{-}$cells $)$
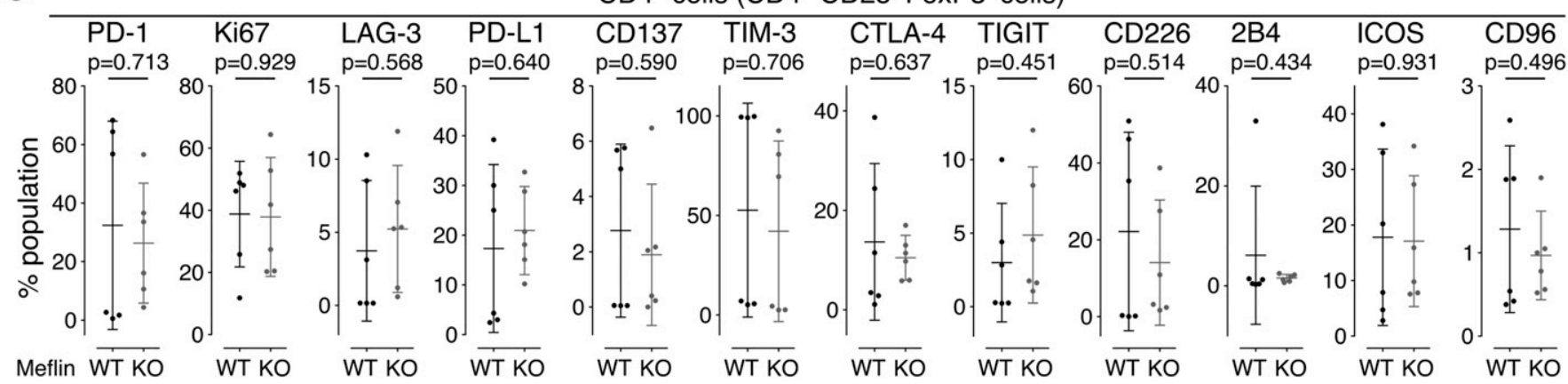

$\mathrm{D}$

$\mathrm{CD}^{+}$cells

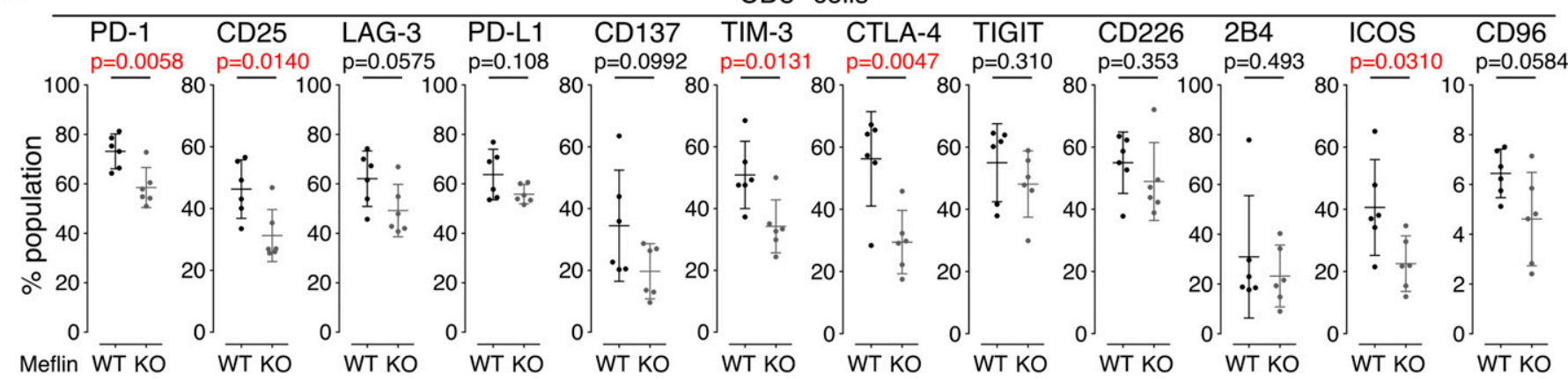

E

Tregs $\left(\mathrm{CD}^{+} \mathrm{CD}^{+} 5^{+} \mathrm{FoxP}^{+}\right.$cells $)$

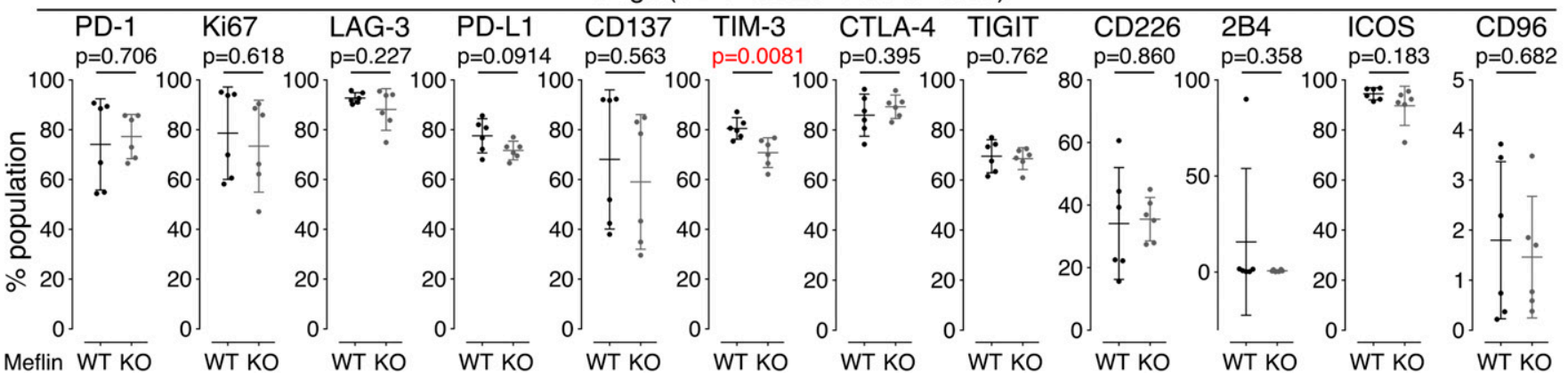

Figure 7. Exploratory analysis of the expression of various markers on TILs isolated from MC-38 tumors developed in wild-type or Meflin-KO mice.

(A, B) MC-38 cells were transplanted into wild-type (WT) or Meflin-KO (KO) mice on day 0, followed by tumor resection and flow cytometric (FCM) analysis on day 9 (A). For FCM analysis, $\mathrm{CD}^{+} \mathrm{T}$ cells were first gated among TILs isolated from the tumors by positive selection, and then these cells were analyzed for expression of the indicated T-cell markers. The graphs on the right show the quantification of $\mathrm{CD} 4^{+}$and $\mathrm{CD} 8^{+} \mathrm{T}$ cells and $\mathrm{CD} 4^{+} \mathrm{CD} 25^{+}$FoxP3 ${ }^{+}$regulatory $\mathrm{T}$ cells (Treg) in TILs isolated from tumors developed in WT and KO mice. (C, D, E) $\mathrm{CD}^{+} \mathrm{CD}^{+} \mathrm{CD} 25^{-}$FoxP3 ${ }^{-}, \mathrm{CD}^{+} \mathrm{CD}^{+}$, and $\mathrm{CD} 4^{+} \mathrm{CD} 25^{+} \mathrm{FoxP} 3^{+} \mathrm{T}$ cells sorted from TILs isolated from tumors developed in wild-type (WT) or Meflin-KO (KO) mice were analyzed for the indicated markers by FCM analysis.

Source data are available for this figure. 

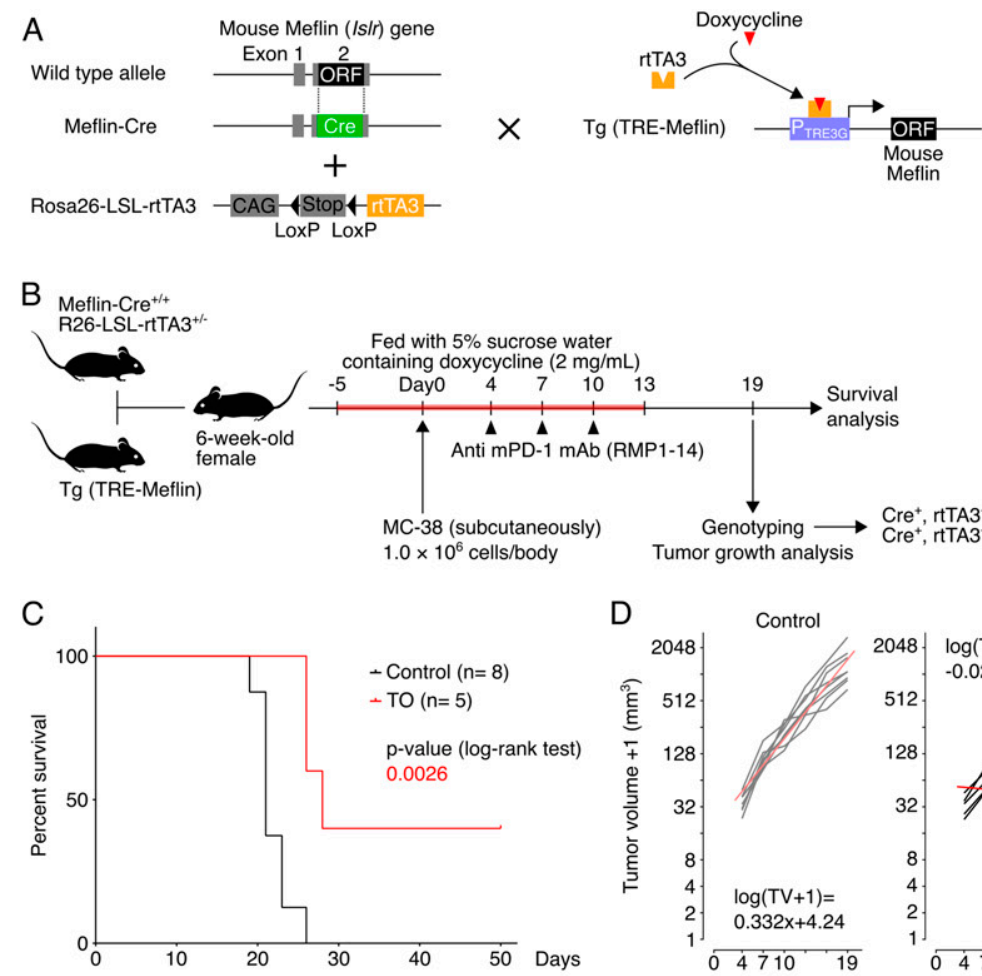

E
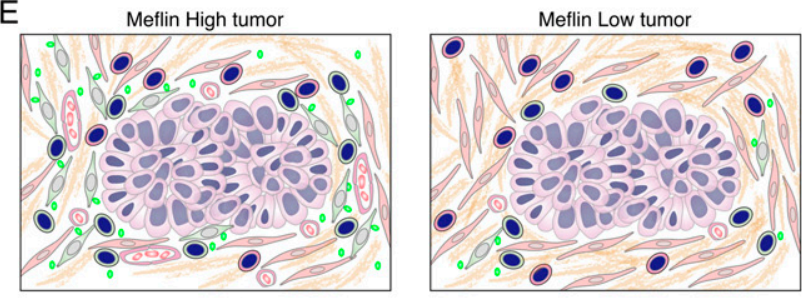

CAF heterogeneity

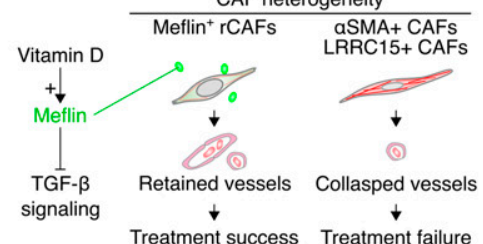

Treatment success Treatment failure

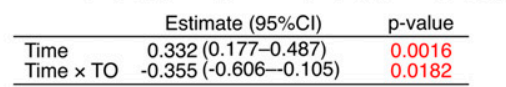

\begin{tabular}{lll} 
Time & $0.332(0.177-0.487)$ & 0.0016 \\
\hline
\end{tabular}

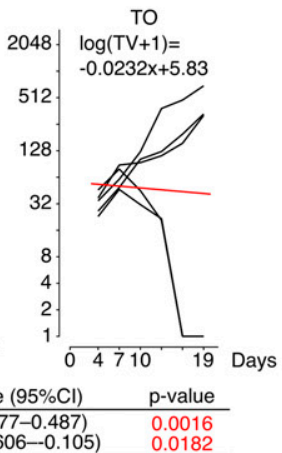

Cre, rtTA3 $^{+}, \mathrm{Tg}^{+}$: Meflin-TO (TO)

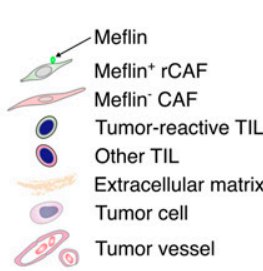

Figure 8. Induced expression of Meflin in cancerassociated fibroblasts (CAFs) improves tumor response to anti-mPD-1 therapy.

(A) A diagram of the generation of the mouse line carrying Meflin-Cre, which constitutively expresses Cre under the control of the Meflin promoter, Rosa26LSL-rtTA3, and Tg (TRE-Meflin) alleles that exhibit induced Meflin expression in Meflin-lineage cells upon doxycycline administration. ORF, open reading frame; CAG, chicken $\beta$-actin promoter; Stop, stop element; rtTA3, the third-generation reverse tetracycline-regulated transactivator; Tg, transgenic; TRE, tetracycline-response element. (B) Meflin-Cre ${ }^{+/+}$; Rosa26-LSL-rtTA3 ${ }^{+/-}$mice were crossed with TREMeflin transgenic mice. The resultant 6-wk-old female mice fed with doxycycline were subcutaneously implanted with MC-38 cells, followed by anti-mPD-1 therapy, genotyping, and tumor analysis. Mice that harbor all of the Meflin-Cre, Rosa26-LSL-rtTA3, and TREMeflin alleles were termed Meflin TO (Tet-on) mice. (C) Survival of control (Meflin-Cre ${ }^{+/-}$; Rosa26-LSLrtTA3 $^{-/-}$; TRE-Meflin) and Meflin TO (Meflin-Cre ${ }^{+/-}$; Rosa26-LSL-rtTA3 ${ }^{+/-}$; TRE-Meflin) mice treated with anti-mPD-1 antibody. (D) Time courses of the volumes of tumors developed in the indicated groups (black and grey lines). For the log transformation of tumor volumes, one was added to every tumor volume. Red lines indicate linear approximations. The table shown under the graphs shows the restricted maximum likelihood estimates of each parameter in a linear mixed-effects model that includes the interactions of time and time and induced Meflin expression (TO) while adding variable effects to the slope and intercept for each individual. (E) Graphical summary and working hypothesis for CAF heterogeneity and its role in immune checkpoint blockade response. Our data demonstrated that infiltration of Meflin ${ }^{+}$CAFs was associated with increased tumor vessel area, which may allow blood and immune cells and macromolecules like antibodies to access the tumor. Source data are available for this figure. and TGF- $\beta$-activated CAFs, suppress antitumor immunity and are associated with ICB therapy failure (Chakravarthy et al, 2018; Mariathasan et al, 2018; Dominguez et al, 2020; Kieffer et al, 2020). We propose that a balance between the immunosuppressive CAFs and Meflin $^{+}$rCAFs is crucial for determining the net response to ICB therapy (Fig 8E).

Given our initial data that Meflin expression in CAFs correlated with the favorable outcomes of NSCLC patients treated with ICB, it was an unexpected finding that the repertoire of TILs was almost comparable between Meflin-high and Meflin-low groups, except for $\mathrm{CD}^{+} \mathrm{T}$ cells. Meflin expression did not affect the infiltration of $\mathrm{CD} 8^{+}$ $T$ cells or regulatory $T$ cells, suggesting that the mechanism of action of Meflin protein and Meflin ${ }^{+}$CAFs may be different from those occurring with other molecules and modalities that directly boost tumor immunity by regulating the interactions of tumor cells with the host tissue. Interestingly, $\mathrm{CD}_{4}{ }^{+} \mathrm{T}$-cell infiltration in the stroma, but not in the tumor parenchyma, correlated with the number of Meflin ${ }^{+}$CAFs in patients with NSCLC. These data may provide a mechanistic clue to the cancer-restraining role of Meflin ${ }^{+}$ rCAFs. Several studies have shown the involvement of $\mathrm{CD}^{+}{ }^{+}$helper $\mathrm{T}$ cells in tumor immunity and response to ICB (Zuazo et al, 2019; Kagamu et al, 2020; Li et al, 2020; Liu et al, 2020), which is distinct from their prominent role in inducing tumor-reactive cytotoxic $T$ cells (Kreiter et al, 2015; Sahin et al, 2017; Borst et al, 2018; Zuazo et al, 2020). Notably, the repertoire of TILs was comparable between tumors developed in WT and Meflin-KO mice in a syngeneic tumor 
model, suggesting that $\mathrm{CD} 4^{+} \mathrm{T}$-cell infiltration depends on the overall function of Meflin ${ }^{+}$CAFs, but not specifically the function of Meflin.

We previously identified BMP7 as a ligand of Meflin and reported that it augments BMP7 signaling, which suppresses TGF- $\beta$ signaling and tissue fibrosis (Hara et al, 2019; Kobayashi et al, 2021). The present data showed that the tumor vessel areas correlated with Meflin expression in CAFs in both human NSCLC tissues and mouse models. Although not proven in the present study, an intriguing hypothesis is that Meflin-mediated suppression of tissue fibrosis or decrease in interstitial pressure facilitates tumor vessel perfusion and therapeutic antibody delivery. In addition, the involvement of Meflin in controlling the enhanced permeability and retention (EPR) effect, which refers to the ability of macromolecules such as anti-PD-1 antibodies to accumulate in the tumor tissue (Matsumura \& Maeda, 1986; Matsumura, 2020), will be a subject of future research.

An appealing feature of Meflin is that none ( $0 \%)$ of the patients with NSCLC in our institution with low Meflin expression in CAFs responded to ICB therapy. These data suggest that the number of Meflin ${ }^{+}$CAFs could be a marker for identifying patients who will not benefit from ICB therapy. The present study also showed that the induced expression of Meflin in CAFs increased the antitumor effect of anti-mPD-1 antibody in a transgenic mouse model. The data implied that the augmentation of Meflin expression in CAFs could be a therapeutic strategy to increase the efficacy of ICB therapy. In relation to this issue, we previously showed that calcipotriol, a vitamin D analog, induced the up-regulation of Meflin expression in CAFs isolated from human pancreatic cancer (Mizutani et al, 2019). Our findings are consistent with a previous study that showed that calcipotriol administration induced changes in the TME with an increase in the delivery of chemotherapeutic agents to the tumor in a pancreatic cancer mouse model (Sherman et al, 2014). Ongoing clinical trials have investigated the use of vitamin D analogs in combination with immune checkpoint inhibitors and chemotherapeutic agents (Gong et al, 2018). It would be interesting to study how Meflin is involved in vitamin D-mediated remodeling of the TME and the increased efficiency of ICB therapy in clinical settings.

In conclusion, we identified a CAF subset marked by Meflin expression and found its prevalence to be associated with a favorable response to ICB therapy in patients with NSCLC and syngeneic tumor mouse models. Induction of Meflin expression in CAFs augmented the tumor response to ICB therapy in mice. Together with other studies that identified CAF subsets that suppress antitumor immunity and are associated with ICB treatment failure, we propose that the heterogeneity of CAFs determines the net response of tumors to ICB therapy.

\section{Materials and Methods}

\section{Subject details}

This study was conducted in accordance with the Declaration of Helsinki and approved by the Ethics Committee of Nagoya University Graduate School of Medicine (approval number 2017-0127-3). We retrospectively enrolled a cohort of patients with NSCLC at Nagoya University Hospital to identify the effects of Meflin ${ }^{+}$CAFs on patient response to ICB monotherapy. The cohort included patients with advanced or recurrent NSCLC who received programmed cell death 1 (PD-1) or programmed cell death 1 ligand 1 (PD-L1) antibody-based ICB monotherapy $(n=132$, Table 1$)$. All patients consented to the Institutional Review Board-approved protocols permitting specimen collection.

\section{Human tumor samples}

98 of 132 patients from the cohort were included for further investigation because of their characteristics described in Fig 4A. 38 of 98 selected patients from the cohort were obtained at the time of surgery, and 60 tumor tissues from the cohort were obtained at the time of diagnostic biopsy or re-biopsy before ICB monotherapy.

\section{Visualization of previously processed single-cell RNA seq dataset}

To visualize Meflin expression in the single-cell RNA sequencing dataset (ArrayExpress accession numbers E-MTAB-6149 and E-MTAB-6653, Lambrechts et al, 2018), we used the web-based visualization tool SCope (https://gbiomed.kuleuven.be/scRNAseqNSCLC, Davie et al, 2018). We also analyzed the dataset via BBrowser (BioTuring, Le et al, 2020 Preprint) to extract data of gene expression in fibroblasts and divide them into ACTA2, IL6, or HLA-DRA-high (normalized value: 1-) or -low/neg (normalized value: $<1)$ groups, followed by processing the data with the R package (v.4.1.2, https:// www.r-project.org/) to obtain density and violin plots using the tidyverse function implemented in the R package (Wickham et al, 2019). Given that the data showed non-normal distribution and heteroscedasticity, we chose a nonparametric Brunner-Munzel test to examine the expression data in each group.

\section{Clinical efficacy analysis}

OS was defined as the time from the start of ICB therapy until death from any cause. PFS was defined as the time from ICB monotherapy initiation until disease progression or death from any cause. Patient follow-up ended when an outcome was recorded or censored as of the database lock on 30 April 2020. Response to ICB was determined according to immunotherapy Response Evaluation Criteria in Solid Tumors (iRECIST) at each time point, which included iCR (complete response), iSD (stable disease), and iPR (partial response), as well as unconfirmed PD (iUPD) and confirmed PD (iCPD). ORR was defined as the ratio of patients who achieved iPR or iCR.

\section{Animals}

All mice were kept in specific pathogen-free conditions in the Division of Experimental Animals, Nagoya University Graduate School of Medicine. All experimental protocols were approved by the Animal Care and Use Committee of Nagoya University Graduate School of Medicine. The generation of an autochthonous lung adenocarcinoma mouse model (KP mice), Meflin-KO mice, and Meflin-Cre mice have been described previously (Maeda et al, 2016; Mizutani et al, 2019; Taki et al, 2020; Hara et al, 2021).

We generated a transgenic mouse line carrying a thirdgeneration tetracycline-response element (TRE)-Meflin to induce Meflin expression in CAFs. To this end, the open reading frame of 
the mouse Meflin gene was inserted into the multiple cloning site of a tetracycline-inducible expression vector pTRE3G (631168; Clontech), followed by microinjection into fertilized eggs of C57BL/ 6 mice (RRID: IMSR_JAX:000664). We established four lines of TREMeflin with germline transmission. We selected one line for further experiments based on the confirmed doxycycline-mediated induction of Meflin expression in mouse embryonic fibroblasts. The TRE-Meflin mice were crossed with Meflin-Cre and Rosa26-CAGsLSL (LoxP-Stop-LoxP)-rtTA3 knock-in mice (hereafter termed R26rtTA3, JAX stock\# 029617, RRID: IMSR_JAX:029617).

Genomic DNA extracted from mouse tails was used for PCRbased genotyping. The primer sequences were as follows: Meflin-KO forward, 5'-GCTGCATTTGAGCTGAGCCTCTGG-3'; Meflin-KO reverse, 5'-AACCCCTTCCTCCTACATAGTTGG-3'; Meflin-Cre forward, 5'-TAGGTGGTATTGGATTCTGGCTGGG-3'; Meflin-Cre reverse, 5'-TTGAAGTAGTCGACGATGTCCTGG-3'; R26-rtTA3 forward, 5'-TACTCAATGGAGTCGGTA TCGAAGGC-3'; R26-rtTA3 reverse, 5'-CCAATACGCAGCCCAGTGTAAA GTGG-3'; TRE-Meflin forward, 5'-GATCGCCTGGAGCAATTCCACAAC-3'; TRE-Meflin reverse, 5'-CTGTTGGCTGACAGGCTCAGTGTGG-3'. The PCR product sizes from Meflin-KO, Meflin-Cre, R26-rtTA3, and TRE-Meflin alleles were 267, 385, 393, and 315 bp, respectively.

\section{Cell lines}

MC-38 (ENH204; Kerafast), a murine colon cancer cell line, and EO771 (94A001; CH3 BioSystems, RRID: CVCL_GR23), a murine breast cancer cell line, were maintained in DMEM (Gibco) and RPMI 1640 (Gibco), respectively, supplemented with $10 \%$ heat-inactivated FBS. All cell lines were routinely screened for mycoplasma contamination by 4 , 6-diamidino-2-phenylindole (DAPI) staining.

\section{Syngeneic tumor studies}

In vivo tumor studies were performed as follows: 6-wk-old WT control and Meflin-KO female mice were inoculated subcutaneously in their right flanks with $1.0 \times 10^{6} \mathrm{MC}-38$ cells suspended in $100 \mu \mathrm{l}$ of PBS or orthotopically in their fourth right mammary fat pads with $5.0 \times 10^{5}$ EO771 cells suspended in $50 \mu \mathrm{l}$ of PBS. The volumes of MC-38 tumors were measured and calculated two to three times per week using the modified ellipsoid formula: $1 / 2 \times$ (length $\times$ width $^{2}$ ). Mice with tumor volumes $>2,000 \mathrm{~mm}^{3}$ were euthanized. Animals whose tumors were ulcerated with bleeding before progression were terminated and included in the study.

\section{In vivo antibody treatment}

To investigate the efficacy of ICB therapy in the MC-38 subcutaneous tumor model, anti-mPD-1 (RMP1-14, RRID: AB_2800578; BioLegend) and isotype control (RTK2758; BioLegend) antibodies were administered intraperitoneally to mice on day 4 , which was $4 \mathrm{~d}$ after tumor inoculation at a dose of $200 \mu \mathrm{g} /$ body, followed by subsequent antibody administration on day 7 and 10 at the same dose. For EO771 orthotopic tumor models, antibody administration was initiated on day 6 after palpable tumors had formed, followed by antibody administration on day 9 and 12 at the same dose via the same route.

\section{Tumor growth/tumor volume analysis}

A linear mixed-effects model was used to examine repeated measurement data to investigate the effect of genotype (G: WT or Meflin-KO), treatment ( $\mathrm{T}$ : control or PD-1), and interaction between $\mathrm{G}$ and $\mathrm{T}$ on tumor volume over time. The following model was used in the analysis:

$\log (T V)=\beta_{0}+b_{0}+\left(\beta_{1}+b_{1}\right) \times$ days $+\beta_{2} \times G+\beta_{3} \times T+\beta_{12} \times$ days $\times G+\beta_{13}$ $\times$ days $\times T+\beta_{123} \times$ days $\times G \times T$

$\left(\mathrm{b}_{0}, \mathrm{~b}_{1}\right) \sim \operatorname{Normal}(0, \Phi), \Phi=\left(\sigma_{\mathrm{B} 0}^{2}, \sigma_{\mathrm{B} 01}, \sigma_{\mathrm{B} 1}^{2}\right)$.

Here, $\beta_{0}, \beta_{1}, \beta_{2}, \beta_{3}, \beta_{12}, \beta_{13}$, and $\beta_{123}$ are the coefficients of the fixed effects. $b_{0}$ is the random effect of the intercept, and $b_{1}$ is the random effect of the slope. $\sigma_{\mathrm{B} 0}^{2}$ is the variance of the individual difference at the baseline, $\sigma_{\mathrm{B} 1}^{2}$ is the variance of the individual difference of the slope, and $\sigma_{\mathrm{B} 01}$ is the covariance of the individual difference of the baseline and the individual difference of the slope. Regression lines were used to fit a linear profile to the time courses of logarithm-transformed tumor volumes in each group. Fitting was performed using customized functions in R v.3.6.3, which integrates software from open-source packages, including Ime4 and ImerTest (Bates et al, 2015; Kuznetsova et al, 2017). Visualization of the growth curves was performed using GraphPad Prism 6.

For EO771 orthotopic tumor models, we measured and analyzed tumor volumes on the day of termination. Because the data showed non-normal distribution and heteroscedasticity, we chose a nonparametric Brunner-Munzel test with permutation to analyze tumor volumes in each group. The effect size was calculated using Cliff's delta statistic method and visualized using functions in R v.3.6.3, which integrates software from an open-source package, including dabestr (Ho et al, 2019).

\section{Tumor processing}

To isolate cells from tumors for FCM analysis, tumors were mechanically dissociated, followed by filtering through 100 and $40 \mu \mathrm{m}$ cell strainers and centrifugation to collect the cells. For qPCR, total RNA was extracted from tumors using TRI-Reagent (Molecular Research Center), following the manufacturer's protocol. To isolate CAFs from tumors, tumors were mechanically minced and digested using the tumor dissociation kit (130-096-730; Miltenyi Biotec) in gentleMACS C-Tubes (130-093-237; Miltenyi Biotec) according to the manufacturer's instructions. Tumor samples were minced and incubated in digestion media at $37^{\circ} \mathrm{C}$ for $30 \mathrm{~min}$ in a gentleMACS Octo dissociator (130-096427; Miltenyi Biotec). After the digestion period, cells were suspended in a cold FACS buffer (0.5\% BSA and 2 mM EDTA in PBS), filtered through $70-\mu \mathrm{m}$ filters, and centrifuged to collect the cells.

\section{Flow cytometry analysis}

FCM staining and analysis were performed using conventional procedures. Cells were washed using a FACS buffer ( $0.5 \%$ BSA and 2 mM EDTA in PBS) and stained with cell-surface antibodies and Fixable Viability Dye eFlour 506 (eBioscience). For the intracellular staining of Foxp3 and Ki-67, cells were fixed and permeabilized using the Foxp3/Transcription Factor Staining Buffer 
Set (eBioscience) according to the manufacturer's instructions, followed by staining with monoclonal antibodies against Foxp3 (1:50 dilution) and Ki-67 (1:100 dilution). After washing, cells were analyzed with a BD LSRFortessa X-20 flow cytometer (BD Biosciences) and Flowjo (TreeStar) software. In this study, the following antimouse antibodies labelled with fluorescent dyes were used: CD3Alexa Fluor (AF) 700 (clone 17A2, RRID: AB_493697), CD4-APC-Fire 750 (clone RM4-4, RRID: AB_2715955), CD8a-Brilliant Violet (BV) 785 (clone 53-6.7, RRID: AB_2562610), CD25-BV605 (clone PC61, RRID: AB_2563059), PD-1-BV421 (clone 29F.1A12, RRID: AB_2561447), PD-L1-APC (clone 10F.9G2, RRID: AB_10612741), CD137-PE (clone 17B5, RRID: AB_2205693), Tim-3-PE-Cyanine7 (clone RMT3-23, RRID: AB_2571932), TIGIT-BV421 (clone 1G9, RRID: AB_2687311), CD96-PE (clone 3.3, RRID: AB_1279389), CD226-BV711 (clone TX42.1, RRID: AB_2715922) (all from BioLegend); FoxP3-PerCP-Cyanine5.5 (clone FJK-16s, RRID: AB_914351), Ki-67-FITC (clone SolA15, RRID: AB_11151689), ICOS-APC (clone 7E.17G9, RRID: AB_2716947) (all from eBioscience); LAG-3-BV711 (clone C9B7W, Cat. no. 563179; BD Bioscience); and 2B4-AF488 (clone 244F4, Cat. no. NBP2-00223AF488; Novus Biologicals).

\section{qPCR}

Total RNA was purified from whole tumors and cultured CAFs using the RNeasy Mini Kit (Cat. no. 74104; QIAGEN) according to the manufacturer's instructions. Purified RNA samples were reversetranscribed using ReverTra Ace (Cat. no. TRT-101; Toyobo) with oligo dT and random primers. qPCR of the generated CDNAs was performed with TaqMan Gene Expression Master Mix (Cat. no. 4369016; Applied Biosystems) using a StepOnePlus thermal cycler (Applied Biosystems). Applied Biosystems synthesizes customized TaqMan probes and primers for the mouse Meflin (Is/r) coding sequence. Cycling conditions were as follows: $50^{\circ} \mathrm{C}$ for $2 \mathrm{~min}, 95^{\circ} \mathrm{C}$ for $10 \mathrm{~min}, 40$ cycles of $95^{\circ} \mathrm{C}$ for $15 \mathrm{~s}$, and $60^{\circ} \mathrm{C}$ for $1 \mathrm{~min}$. The data were analyzed using the $2^{-\Delta \Delta C t}$ method and normalized to Col1a1 (Mm00801666_g1).

\section{RNA in situ hybridization}

To detect single mRNA molecules, an RNA in situ hybridization (ISH) assay based on RNAscope technology (Advanced Cell Diagnostics, ACD) was performed on formalin-fixed and paraffin-embedded (FFPE) human and mouse tissue samples and fixed cultured cells. Sample preparation was performed according to the manufacturer's protocol for RNAscope on tissue samples (ACD, document number 322452) or the technical note for fixed cultured cells (ACD, document number MK-50010). ISH was performed according to the protocol of the RNAscope $2.5 \mathrm{HD}$ Detection Reagent-BROWN (ACD, Cat. no. 322310), the 2.5 HD Duplex Detection Kit (ACD, Cat. no. 322500), or Multiplex Fluorescent Reagent Kit v2 (ACD, Cat. no. 323100). Briefly, FFPE sections were deparaffinized in xylene and $100 \%$ ethanol and dried completely for $5 \mathrm{~min}$ at room temperature (RT). After incubation with $\mathrm{H}_{2} \mathrm{O}_{2}$ solution for 10 min at RT, slides were treated in a boiling target retrieval solution $\left(>98^{\circ} \mathrm{C}\right)$ for $15 \mathrm{~min}$, washed in distilled water, dehydrated in $100 \%$ ethanol, and dried completely. Finally, the slides were incubated with Protease Plus (ACD) at $40^{\circ} \mathrm{C}$ for $30 \mathrm{~min}$. After washing in distilled water, slides were incubated with the relevant probes at $40^{\circ} \mathrm{C}$ for $2 \mathrm{~h}$, followed by amplification through sequential amplification using AMP-1 to AMP-6 reagents $(A C D)$. Staining was visualized with 3,3-diaminobenzidine (DAB) followed by counterstaining with hematoxylin. To detect the expression of more than two mRNAs on the same slides using bright field microscopy, we used the 2.5 HD Duplex Detection Kit (Fast Green and Fast Red, ACD), staining with alkaline phosphatase (ALP), and $D A B$ followed by counterstaining with hematoxylin. To detect the expression of more than two mRNAs on the same slides by fluorescent microscopy, we used Multiplex Fluorescent Reagent Kit V2 (ACD), staining with fluorophores TSA (tyramide signal amplification)- cyanine 3 and - cyanine 5 (AKOYA Bioscience), followed by counterstaining with DAPI. In this study, the following six different probes against genes of interest were used: Hs-ISLR (human Meflin) (NM_005545.3, region 275-1322; Cat. no. 455481), Mm-Islr (mouse Meflin) (NM_012043.4, region 763-1690; Cat. no. 450041), Hs-ACTA2 (NM_001613.2, region 10-1341; Cat. no. 311811), Hs-PDGFRA (NM_006206.4, region 844-1774; Cat. no. 604481), and Hs-PDPN (NM_001006625.1, region 911-2045; Cat. no. 539751).

\section{Assessment of ISH staining}

To assess Meflin expression in the stroma, we first counted total stromal cells other than immune cells including macrophages identified by their morphology. The Meflin-positive cells were then counted and divided by the total stromal cells to calculate the percentage in the stroma. When cells had four and more dots or had any clusters of ISH signals, we considered them positive. We semi-quantitatively scored the expression of Meflin in each specimen (Fig 1C) or each patient (Figs 3B and 4B) according to the percentage of Meflin-positive cells. Specifically, $0 \%$ and $1-5 \%$ stromal cell expressing Meflin were combined into the score " $<5$ " which referred to " 0 " in Fig $1 C$; thereafter, we scored 5-10\% as " 5 ," $10-15 \%$ as "10," $15-20 \%$ as "15" and so forth. For assessing KP mice specimens (Fig 1D), quantitative values were used except for INV to clarify the difference between TA and PIL.

\section{IHC assay using the Opal-IHC kit and immunofluorescent staining combined with RNAscope}

For IHC, FFPE tissue sections were deparaffinized and subjected to antigen retrieval using a target retrieval solution (Dako or Novocastra) at $\mathrm{pH}$ 6, 7, or 9 for 30 min in an electric kettle, followed by IHC using conventional procedures. For multiplex immunofluorescent (IF) staining of TILs, the Opal 7 Tumor Infiltrating Lymphocyte Kit (Cat. no. OP7TL3001KT; Akoya Bioscience) was used to stain CD4, CD8, CD20, FoxP3, CD45RO, and pan-cytokeratin, according to the manufacturer's instructions. The sections were mounted using PermaFluor Aqueous Mounting Medium (Cat. no. TA-030-FM; Thermo Fisher Scientific), followed by scanning using a Vectra slide scanner (Akoya Bioscience). Five randomly selected multispectral high-powered field images of each section were captured using an automated imaging system (Vectra ver. 3.0, Akoya Bioscience) and loaded into usertrainable image analysis software (InForm, Akoya Bioscience), which allows the automatic recognition of tissue regions and individual cells to perform cell classification and phenotyping.

For the combined detection of mRNAs by ISH and proteins by IF, we first stained FFPE sections by ISH with the fluorophore TSACyanine 5 for visualization. The ISH-stained slides were washed 
with PBS twice and incubated with blocking buffer containing 10\% serum (of the host from which secondary antibodies were derived) for 30 min at RT, followed by incubation with primary antibodies diluted in PBS at $4^{\circ} \mathrm{C}$ overnight. After washing with PBS, the slides were incubated with Alexa Fluor 488/594-conjugated secondary antibodies (Thermo Fisher Scientific) for $30 \mathrm{~min}$ at RT, followed by incubation with DAPI for $30 \mathrm{~s}$ at RT and mounting with PermaFluor aqueous mounting medium.

For proteins for which corresponding antibodies that work for IF after performing ISH are unavailable, such as LCA and podoplanin (Fig S2A and B), we first performed IF using Opal fluorophores (NEL810001KT; Akoya Biosciences) according to the manufacturer's instructions. Briefly, FFPE sections were deparaffinized, followed by antigen retrieval in AR6 or AR9 (AR600250ML, AR900250ML; Akoya Biosciences) for $15 \mathrm{~min}$ at $98^{\circ} \mathrm{C}$. After blocking with Antibody Diluent/Blocking (ARD1001EA; Akoya Biosciences), the sections were incubated with primary antibodies for $2 \mathrm{~h}$ at RT or overnight at $4^{\circ} \mathrm{C}$, washed three times in Tris-buffered saline $(150 \mathrm{mM} \mathrm{NaCl}$ and $25 \mathrm{mM}$ Tris- $\mathrm{HCl}$ ) with $0.05 \%$ polyoxyethylene (20) sorbitan monolaurate (Tween 20) (TBST), and incubated in Opal Polymer HRP secondary antibody (ARH1001EA; Akoya Biosciences) for 10 min at RT. After three washes in TBST, Opal 570 in 1X Plus Amplification Diluent (1: 125) was added and reacted for 10 min at RT. After three washes in TBST, we performed ISH using RNAscope Multiplex Fluorescent Reagent Kit v2 (323100; ACD) according to the manufacturer's instructions. For mRNA retrieval and the removal of antibody-HRP complexes, the slides were immersed in $1 \mathrm{X}$ Target Retrieval Buffer using a pressure cooker (Cat. no. SRMP300; Panasonic) for 15 min, followed by washing the sections in distilled water and dehydrating them in $100 \%$ ethanol. After drying the slides for $5 \mathrm{~min}$ at $60^{\circ} \mathrm{C}$, they were treated with Proteinase Plus (Cat. no. 322331; ACD) for 30 min at $40^{\circ} \mathrm{C}$. The sections were washed once in deionized water, incubated with target probes for $2 \mathrm{~h}$ at $40^{\circ} \mathrm{C}$, washed twice in $1 \times$ wash buffer, and then incubated in amplification reagents (AMP1-3), followed by HRP-C1 Reagent (Cat. no. 323104; ACD). The signals were amplified with a TSA-Plus Cyanine 5 System (Cat. no. NEL745001KT; PerkinElmer), followed by mounting the sections with PermaFluor Aqueous Mounting Medium. Fluorescence was examined using an inverse immunofluorescence microscope BZ-X710 (Keyence) with optical sectioning.

The following antibodies were used in the present study: mouse monoclonal anti-E-cadherin (clone 36, Cat. no. 610181, dilution 1:500; BD Biosciences), mouse monoclonal anti-human CD31 (clone JC70A, Cat. no. M0823, dilution 1:250; Dako), mouse monoclonal antiLCA (clone 2B11 + PD7/26, Cat. no. IR751; Dako), rabbit polyclonal anti-Col1a1 (Cat. no. NB600-408, dilution 1:500; Novus), mouse monoclonal anti- $\alpha$-SMA (clone 1A4, Cat. no. M0851, dilution 1:500; Dako), mouse monoclonal anti-Podoplanin (clone D2-40, Cat. no. ab77854, dilution 1:100; Abcam), and rat monoclonal anti-mouse CD31 (clone SZ31, Cat. no. DIA-310, dilution 1:100; Dianova).

\section{Statistical analysis}

We used GraphPad Prism 6 or R v.4.1.2 for statistical analysis. Patient characteristics and binary outcomes were compared between the two groups using Fisher's exact test or the Mann-Whitney $U$ test. Survival was analyzed using the Kaplan-Meier approach and the log-rank Mantel-Cox test, as well as the Cox proportional hazards regression model. Variables with $P$-values $<0.2$ on the univariate Cox models were included in the multivariate analyses. Also, Meflin expression status, PD-L1 TPS, and the variables with $P$-values $<0.1$ on the analyses of patient characteristics were included in the final model, irrespective of their statistical significance. The magnitudes of the associations were summarized using hazard ratios with $95 \%$ confidence intervals (Cls). To evaluate the predictive value for responders, a receiver operating characteristic curve (ROC) for discrete variables was created by plotting the true-positive rate against the false-positive rate at each threshold setting. The area under the curve (AUC) shown in the plot summarizes the performance of discrete variables. The cut-off value of discrete variables in which the sum of sensitivity and specificity was the maximum was detected. For murine experiments, all data are representative of at least two to three independent experiments with three to six mice in each in vivo experiment. The data were expressed as means with $95 \%$ Cls unless otherwise specified. The relationships between groups were compared using a two-tailed unpaired $t$ test with Welch's correction unless otherwise specified. For multiple testing, the Holm-Bonferroni method was employed. Survival was analyzed using the Kaplan-Meier approach and the log-rank Mantel-Cox test. Statistical significance was set at $P<0.05$.

\section{Data Availability}

This manuscript does not have large-scale data sets to deposit to the public databases.

\section{Supplementary Information}

Supplementary Information is available at https://doi.org/10.26508/lsa 202101230.

\section{Acknowledgements}

We thank Kaori Ushida and Kozo Uchiyama (Nagoya University) for their support in immunostaining and tissue preparation and the staff of the Division of Experimental Animals (Nagoya University) for their support in animal experiments. We thank Editage (http://www.editage.com) for editing and reviewing this manuscript for English language. This work was supported by a Grant-in-Aid for Scientific Research (B) (18H02638 to A Enomoto, $20 \mathrm{H} 03467$ to M Takahashi, $20 \mathrm{H} 03528$ to $\mathrm{Y}$ Ando) and a Grant-in-Aid for Research Activity Startup (20K22807 to Y Miyai) commissioned by Japan Society for the Promotion of Science; Nagoya University Hospital Funding for Clinical Research (to A Enomoto); AMED-CREST (Japan Agency for Medical Research and Development, Core Research for Evolutional Science and Technology; 20gm0810007h0105 and 20gm1210009s0102 to A Enomoto), the Project for Cancer Research and Therapeutic Evolution (P-CREATE) from AMED (20cm0106377h0001 to A Enomoto), and Aichi Cancer Research Foundation (to Y Miyai).

\section{Author Contributions}

Y Miyai: conceptualization, data curation, formal analysis, funding acquisition, validation, investigation, visualization, methodology, and writing-original draft, review, and editing. 
D Sugiyama: data curation, formal analysis, and methodology.

T Hase: resources.

N Asai: resources.

T Taki: resources and investigation.

K Nishida: formal analysis.

T Fukui: resources.

TF Chen-Yoshikawa: resources.

H Kobayashi: resources.

S Mii: resources, investigation, and writing-review and editing.

Y Shiraki: resources and investigation.

Y Hasegawa: resources.

H Nishikawa: resources and supervision.

$Y$ Ando: resources, supervision, and funding acquisition.

M Takahashi: resources, supervision, and funding acquisition.

A Enomoto: supervision, funding acquisition, project administration, and writing-original draft, review, and editing.

\section{Conflict of Interest Statement}

Y Miyai is the primary inventor on a pending patent PCT/JP2019/004521 related to the current work belonging to Nagoya University. T Hase has received honoraria from AstraZeneca, Ono Pharmaceutical, Chugai Pharmaceutical, and Bristol-Myers Squibb and received research funding from Boehringer Ingelheim and Taiho Pharmaceutical outside the scope of this work. Y Hasegawa has received honoraria from AstraZeneca, Ono Pharmaceutical, Chugai Pharmaceutical, and Taiho Pharmaceutical outside the scope of this work. H Nishikawa received honoraria and research funding from Ono Pharmaceutical, Chugai Pharmaceutical, MSD, and Bristol-Myers Squibb, and research funding from Taiho Pharmaceutical, Daiichi-Sankyo, Kyowa Kirin, Zenyaku Kogyo, Oncolys BioPharma, Debiopharma, Asahi-Kasei, Sysmex, Fujifilm, SRL, Astellas Pharmaceutical, Sumitomo Dainippon Pharma, and BD Japan outside of this study. Y A has received honoraria from Chugai Pharmaceutical and received research funding from Novartis, Ono Pharmaceutical, Chugai Pharmaceutical, Bayer Yakuhin, and Yakult Honsha outside the scope of this work. The other authors declare no competing interests.

\section{References}

Baker AT, Abuwarwar MH, Poly L, Wilkins S, Fletcher AL (2021) Cancerassociated fibroblasts and T cells: From mechanisms to outcomes. J Immunol 206: 310-320. doi:10.4049/jimmunol.2001203

Barrett RL, Puré E (2020) Cancer-associated fibroblasts and their influence on tumor immunity and immunotherapy. Elife 9: e57243. doi:10.7554/ eLife. 57243

Bates D, Mächler M, Bolker B, Walker S (2015) Fitting linear mixed-effects models Usinglme4. J Stat Softw 67: 1-48. doi:10.18637/jss.v067.01

Borst J, Ahrends T, Babała N, Melief CJM, Kastenmüller W (2018) CD4+ T cell help in cancer immunology and immunotherapy. Nat Rev Immunol 18: 635-647. doi:10.1038/s41577-018-0044-0

Carbognin L, Pilotto S, Milella M, Vaccaro V, Brunelli M, Caliò A, Cuppone F, Sperduti I, Giannarelli D, Chilosi M, et al (2015) Differential activity of nivolumab, pembrolizumab and MPDL3280A according to the tumor expression of programmed death-ligand-1 (PD-L1): Sensitivity analysis of trials in melanoma, lung and genitourinary cancers. PLOS One 10: e0130142. doi:10.1371/journal.pone.0130142

Carbone DP, Reck M, Paz-Ares L, Creelan B, Horn L, Steins M, Felip E, van den Heuvel MM, Ciuleanu TE, Badin F, et al (2017) First-line nivolumab in stage IV or recurrent non-small-cell lung cancer. N Engl I Med 376: 2415-2426. doi:10.1056/NEJMoa1613493
Chakravarthy A, Khan L, Bensler NP, Bose P, De Carvalho DD (2018) TGF$\beta$-associated extracellular matrix genes link cancer-associated fibroblasts to immune evasion and immunotherapy failure. Nat Commun 9: 4692. doi:10.1038/s41467-018-06654-8

Costa A, Kieffer Y, Scholer-Dahirel A, Pelon F, Bourachot B, Cardon M, Sirven P, Magagna I, Fuhrmann L, Bernard C, et al (2018) Fibroblast heterogeneity and immunosuppressive environment in human breast cancer. Cancer Cell 33: 463-479.e10. doi:10.1016/ j.ccell.2018.01.011

Davie K, Janssens J, Koldere D, De Waegeneer M, Pech U, Kreft $七$, Aibar S, Makhzami S, Christiaens V, Bravo González-Blas C, et al (2018) A singlecell transcriptome atlas of the aging Drosophila brain. Cell 174: 982-998.e20. doi:10.1016/j.cell.2018.05.057

Dominguez CX, Müller S, Keerthivasan S, Koeppen H, Hung J, Gierke S, Breart B, Foreman O, Bainbridge TW, Castiglioni A, et al (2020) Single-cell RNA sequencing reveals stromal evolution into LRRC15+ myofibroblasts as a determinant of patient response to cancer immunotherapy. Cancer Discov 10: 232-253. doi:10.1158/ 2159-8290.CD-19-0644

Dow LE, Nasr Z, Saborowski M, Ebbesen SH, Manchado E, Tasdemir N, Lee T, Pelletier J, Lowe SW (2014) Conditional reverse tet-transactivator mouse strains for the efficient induction of TRE-regulated transgenes in mice. PLoS One 9: e95236. doi:10.1371/journal.pone.0095236

DuPage M, Dooley AL, Jacks T (2009) Conditional mouse lung cancer models using adenoviral or lentiviral delivery of Cre recombinase. Nat Protoc 4: 1064-1072. doi:10.1038/nprot.2009.95

Elyada E, Bolisetty M, Laise P, Flynn WF, Courtois ET, Burkhart RA, Teinor JA, Belleau P, Biffi G, Lucito MS, et al (2019) Cross-species single-cell analysis of pancreatic ductal adenocarcinoma reveals antigenpresenting cancer-associated fibroblasts. Cancer Discov 9: 1102-1123. doi:10.1158/2159-8290.CD-19-0094

Gong J, Hendifar A, Tuli R, Chuang J, Cho M, Chung V, Li D, Salgia R (2018) Combination systemic therapies with immune checkpoint inhibitors in pancreatic cancer: Overcoming resistance to single-agent checkpoint blockade. Clin Transl Med 7: 32. doi:10.1186/s40169-0180210-9

Gros A, Parkhurst MR, Tran E, Pasetto A, Robbins PF, Ilyas S, Prickett TD, Gartner JJ, Crystal JS, Roberts IM, et al (2016) Prospective identification of neoantigen-specific lymphocytes in the peripheral blood of melanoma patients. Nat Med 22: 433-438. doi:10.1038/nm.4051

Gros A, Robbins PF, Yao X, Li YF, Turcotte S, Tran E, Wunderlich JR, Mixon A, Farid S, Dudley ME, et al (2014) PD-1 identifies the patient-specific $\mathrm{CD}^{+}$tumor-reactive repertoire infiltrating human tumors. J Clin Invest 124: 2246-2259. doi:10.1172/JCI73639

Hara A, Kato K, Ishihara T, Kobayashi H, Asai N, Mii S, Shiraki Y, Miyai Y, Ando R, Mizutani Y, et al (2021) Meflin defines mesenchymal stem cells and/or their early progenitors with multilineage differentiation capacity. Genes Cells 26: 495-512. doi:10.1111/gtc.12855

Hara A, Kobayashi H, Asai N, Saito S, Higuchi T, Kato K, Okumura T, Bando YK, Takefuji M, Mizutani Y, et al (2019) Roles of the mesenchymal stromal/ stem cell marker meflin in cardiac tissue repair and the development of diastolic dysfunction. Circ Res 125: 414-430. doi:10.1161/ CIRCRESAHA.119.314806

Ho J, Tumkaya T, Aryal S, Choi H, Claridge-Chang A (2019) Moving beyond P values: Data analysis with estimation graphics. Nat Methods 16: 565-566. doi:10.1038/s41592-019-0470-3

House IG, Savas P, Lai J, Chen AXY, Oliver AJ, Teo ZL, Todd KL, Henderson MA, Giuffrida L, Petley EV, et al (2020) Macrophage-derived CXCL9 and CXCL10 are required for antitumor immune responses following immune checkpoint blockade. Clin Cancer Res 26: 487-504. doi:10.1158/1078-0432.CCR-19-1868

Ichihara R, Shiraki Y, Mizutani Y, lida T, Miyai Y, Esaki N, Kato A, Mii S, Ando R, Hayashi M, et al (2022) Matrix remodeling-associated protein 8 is a 
marker of a subset of cancer-associated fibroblasts in pancreatic cancer. Pathol Int doi:10.1111/pin.131980nline ahead of print.

Kagamu H, Kitano S, Yamaguchi O, Yoshimura K, Horimoto K, Kitazawa M, Fukui K, Shiono A, Mouri A, Nishihara F, et al (2020) CD4+ T-cell immunity in the peripheral blood correlates with response to antiPD-1 therapy. Cancer Immunol Res 8: 334-344. doi:10.1158/2326 6066.CIR-19-0574

Kalluri R (2016) The biology and function of fibroblasts in cancer. Nat Rev Cancer 16: 582-598. doi:10.1038/nrc.2016.73

Kieffer Y, Hocine HR, Gentric G, Pelon F, Bernard C, Bourachot B, Lameiras S, Albergante L, Bonneau C, Guyard A, et al (2020) Single-cell analysis reveals fibroblast clusters linked to immunotherapy resistance in cancer. Cancer Discov 10: 1330-1351. doi:10.1158/21598290.CD-19-1384

Kobayashi H, Enomoto A, Woods SL, Burt AD, Takahashi M, Worthley DL (2019) Cancer-associated fibroblasts in gastrointestinal cancer. Nat Rev Gastroenterol Hepatol 16: 282-295. doi:10.1038/s41575-019-0115-0

Kobayashi H, Gieniec KA, Wright JA, Wang T, Asai N, Mizutani Y, Lida T, Ando R, Suzuki N, Lannagan TRM, et al (2021) The balance of stromal BMP signaling mediated by GREM1 and ISLR drives colorectal carcinogenesis. Gastroenterology 160: 1224-1239.e30. doi:10.1053/ j.gastro.2020.11.011

Kreiter S, Vormehr M, van de Roemer N, Diken M, Löwer M, Diekmann J, Boegel S, Schrörs B, Vascotto F, Castle JC, et al (2015) Mutant MHC class II epitopes drive therapeutic immune responses to cancer. Nature 520: 692-696. doi:10.1038/nature14426

Kumagai S, Togashi Y, Kamada T, Sugiyama E, Nishinakamura H, Takeuchi Y, Vitaly K, Itahashi K, Maeda Y, Matsui S, et al (2020b) The PD-1 expression balance between effector and regulatory $T$ cells predicts the clinical efficacy of PD-1 blockade therapies. Nat Immunol 21: 1346-1358. doi:10.1038/s41590-020-0769-3

Kumagai S, Togashi Y, Sakai C, Kawazoe A, Kawazu M, Ueno T, Sato E, Kuwata T, Kinoshita T, Yamamoto M, et al (2020a) An oncogenic alteration creates a microenvironment that promotes tumor progression by conferring a metabolic advantage to regulatory T cells. Immunity 53: 187-203.e8. doi:10.1016/j.immuni.2020.06.016

Kuznetsova A, Brockhoff PB, Christensen RHB (2017) ImerTest package: Tests in linear mixed effects models. J Stat Softw 82: 1-26. doi:10.18637/ jss.v082.i13

Lambrechts D, Wauters E, Boeckx B, Aibar S, Nittner D, Burton O, Bassez A, Decaluwé H, Pircher A, Van den Eynde K, et al (2018) Phenotype molding of stromal cells in the lung tumor microenvironment. Nat Med 24: 1277-1289. doi:10.1038/s41591-018-0096-5

Le T, Phan T, Pham M, Tran D, Lam L, Nguyen T, Truong T, Vuong H, Luu T, Phung N, et al (2020) BBrowser: Making single-cell data easily accessible. BioRxiv doi:10.1101/2020.12.11.414136Preprint posted December 11, 2020.

Lee JJ, Perera RM, Wang H, Wu DC, Liu XS, Han S, Fitamant J, Jones PD, Ghanta KS, Kawano S, et al (2014) Stromal response to Hedgehog signaling restrains pancreatic cancer progression. Proc Natl Acad Sci U S A 111: E3091-E3100. doi:10.1073/pnas.1411679111

Li S, Liu M, Do MH, Chou C, Stamatiades EG, Nixon BG, Shi W, Zhang X, Li P, Gao $\mathrm{S}$, et al (2020) Cancer immunotherapy via targeted TGF- $\beta$ signalling blockade in TH cells. Nature 587: 121-125. doi:10.1038/s41586-0202850-3

Liu M, Kuo F, Capistrano KJ, Kang D, Nixon BG, Shi W, Chou C, Do MH, Stamatiades EG, Gao S, et al (2020) TGF- $\beta$ suppresses type 2 immunity to cancer. Nature 587: 115-120. doi:10.1038/s41586-020-2836-1

Lu S, Stein JE, Rimm DL, Wang DW, Bell JM, Johnson DB, Sosman JA, Schalper $\mathrm{KA}$, Anders RA, Wang $\mathrm{H}$, et al (2019) Comparison of biomarker modalities for predicting response to PD-1/PD-L1 checkpoint blockade: A systematic review and meta-analysis. JAMA Oncol 5: 1195-1204. doi:10.1001/jamaoncol.2019.1549
Maeda K, Enomoto A, Hara A, Asai N, Kobayashi T, Horinouchi A, Maruyama S, Ishikawa Y, Nishiyama T, Kiyoi H, et al (2016) Identification of meflin as a potential marker for mesenchymal stromal cells. Sci Rep 6: 22288. doi:10.1038/srep22288

Mager LF, Burkhard R, Pett N, Cooke NCA, Brown K, Ramay H, Paik S, Stagg J, Groves RA, Gallo M, et al (2020) Microbiome-derived inosine modulates response to checkpoint inhibitor immunotherapy. Science 369: 1481-1489. doi:10.1126/science.abc3421

Mariathasan S, Turley SJ, Nickles D, Castiglioni A, Yuen K, Wang Y, Kadel EE, Koeppen H, Astarita JL, Cubas R, et al (2018) TGF $\beta$ attenuates tumour response to $\mathrm{PD}-\mathrm{L} 1$ blockade by contributing to exclusion of $\mathrm{T}$ cells. Nature 554: 544-548. doi:10.1038/nature25501

Matsumura Y (2020) Cancer stromal targeting therapy to overcome the pitfall of EPR effect. Adv Drug Deliv Rev 154: 142-150. doi:10.1016/ j.addr.2020.07.003

Matsumura Y, Maeda H (1986) A new concept for macromolecular therapeutics in cancer chemotherapy: Mechanism of tumoritropic accumulation of proteins and the antitumor agent smancs. Cancer Res 46: 6387-6392.

Miyai Y, Esaki N, Takahashi M, Enomoto A (2020) Cancer-associated fibroblasts that restrain cancer progression: Hypotheses and perspectives. Cancer Sci 111: 1047-1057. doi:10.1111/cas.14346

Mizutani Y, Kobayashi H, lida T, Asai N, Masamune A, Hara A, Esaki N, Ushida K, Mii S, Shiraki Y, et al (2019) Meflin-positive cancer-associated fibroblasts inhibit pancreatic carcinogenesis. Cancer Res 79: 5367-5381. doi:10.1158/0008-5472.CAN-19-0454

Nakahara Y, Hashimoto N, Sakamoto K, Enomoto A, Adams TS, Yokoi T, Omote N, Poli S, Ando A, Wakahara K, et al (2021) Fibroblasts positive for meflin have anti-fibrotic properties in pulmonary fibrosis. Eur Respir J 58: 2003397. doi:10.1183/13993003.03397-2020

Öhlund D, Handly-Santana A, Biffi G, Elyada E, Almeida AS, Ponz-Sarvise M, Corbo V, Oni TE, Hearn SA, Lee EJ, et al (2017) Distinct populations of inflammatory fibroblasts and myofibroblasts in pancreatic cancer. J Exp Med 214: 579-596. doi:10.1084/jem.20162024

Özdemir BC, Pentcheva-Hoang T, Carstens JL, Zheng X, Wu CC, Simpson TR, Laklai H, Sugimoto $H$, Kahlert C, Novitskiy SV, et al (2014) Depletion of carcinoma-associated fibroblasts and fibrosis induces immunosuppression and accelerates pancreas cancer with reduced survival. Cancer Cell 25: 719-734. doi:10.1016/ j.ccr.2014.04.005

Piersma B, Hayward MK, Weaver VM (2020) Fibrosis and cancer: A strained relationship. Biochim Biophys Acta Rev Cancer 1873: 188356. doi:10.1016/j.bbcan.2020.188356

Rhim AD, Oberstein PE, Thomas DH, Mirek ET, Palermo CF, Sastra SA, Dekleva EN, Saunders T, Becerra CP, Tattersall IW, et al (2014) Stromal elements act to restrain, rather than support, pancreatic ductal adenocarcinoma. Cancer Cell 25: 735-747. doi:10.1016/j.ccr.2014.04.021

Rizvi NA, Hellmann MD, Snyder A, Kvistborg P, Makarov V, Havel JJ, Lee W, Yuan J, Wong P, Ho TS, et al (2015) Cancer immunology. Mutational landscape determines sensitivity to PD-1 blockade in non-small cell lung cancer. Science 348: 124-128. doi:10.1126/science.aaa1348

Sahin U, Derhovanessian E, Miller M, Kloke BP, Simon P, Löwer M, Bukur V, Tadmor AD, Luxemburger U, Schrörs B, et al (2017) Personalized RNA mutanome vaccines mobilize poly-specific therapeutic immunity against cancer. Nature 547: 222-226. doi:10.1038/nature23003

Seymour L, Bogaerts J, Perrone A, Ford R, Schwartz LH, Mandrekar S, Lin NU, Litière S, Dancey J, Chen A, et al (2017) iRECIST: Guidelines for response criteria for use in trials testing immunotherapeutics. Lancet Oncol 18: e143-e152. doi:10.1016/S1470-2045(17)30074-8

Shen X, Zhao B (2018) Efficacy of PD-1 or PD-L1 inhibitors and PD-L1 expression status in cancer: Meta-analysis. BMJ 362: K3529. doi:10.1136/bmj.k3529 
Sherman MH, Yu RT, Engle DD, Ding N, Atkins AR, Tiriac H, Collisson EA, Connor F, Van Dyke T, Kozlov S, et al (2014) Vitamin D receptor-mediated stromal reprogramming suppresses pancreatitis and enhances pancreatic cancer therapy. Cell 159: 80-93. doi:10.1016/j.cell.2014.08.007

Shin K, Lim A, Zhao C, Sahoo D, Pan Y, Spiekerkoetter E, Liao JC, Beachy PA (2014) Hedgehog signaling restrains bladder cancer progression by eliciting stromal production of urothelial differentiation factors. Cancer Cell 26: 521-533. doi:10.1016/j.ccell.2014.09.001

Smith SM, Wachter K, Burris HA, Schilsky RL, George DJ, Peterson DE, Johnson ML, Markham MJ, Mileham KF, Beg MS, et al (2021) Clinical cancer advances 2021: ASCO[R8S2Q1M7]s report on progress against cancer. J Clin Oncol 39: 1165-1184. doi:10.1200/JCO.20.03420

Takahashi M, Kobayashi H, Mizutani Y, Hara A, lida T, Miyai Y, Asai N, Enomoto A (2021) Roles of the mesenchymal stromal/stem cell marker Meflin/Islr in cancer fibrosis. Front Cell Dev Biol 9: 749924. doi:10.3389/fcell.2021.749924

Taki T, Shiraki Y, Enomoto A, Weng L, Chen C, Asai N, Murakumo Y, Yokoi K, Takahashi M, Mii S (2020) CD109 regulates in vivo tumor invasion in lung adenocarcinoma through TGF- $\beta$ signaling. Cancer Sci 111: 4616-4628. doi:10.1111/cas.14673

Wickham H, Averick M, Bryan J, Chang W, McGowan L, François R, Grolemund G, Hayes A, Henry L, Hester J, et al (2019) Welcome to the tidyverse. J Open Source Softw 4: 1686. doi:10.21105/joss.01686
Xiao Z, Mayer AT, Nobashi TW, Gambhir SS (2020) ICOS is an indicator of Tcell-mediated response to cancer immunotherapy. Cancer Res 80: 3023-3032. doi:10.1158/0008-5472.CAN-19-3265

Zheng X, Fang Z, Liu X, Deng S, Zhou P, Wang X, Zhang C, Yin R, Hu H, Chen X, et al (2018) Increased vessel perfusion predicts the efficacy of immune checkpoint blockade. J Clin Invest 128: 2104-2115. doi:10.1172/ JCl96582

Zuazo M, Arasanz H, Fernández-Hinojal G, García-Granda MJ, Gato M, Bocanegra A, Martínez M, Hernández B, Teijeira L, Morilla I, et al (2019) Functional systemic CD4 immunity is required for clinical responses to PD-L1/PD-1 blockade therapy. EMBO Mol Med 11: e10293. doi:10.15252/emmm.201910293

Zuazo M, Arasanz H, Bocanegra A, Fernandez G, Chocarro L, Vera R, Kochan G, Escors D (2020) Systemic CD4 immunity as a key contributor to PD-L1/ PD-1 blockade immunotherapy efficacy. Front Immunol 11: 3087. doi:10.3389/fimmu.2020.586907

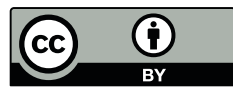

License: This article is available under a Creative Commons License (Attribution 4.0 International, as described at https://creativecommons.org/ licenses/by/4.0/). 